F. BÉGUIN

C. BONATTI

J. L. VIEITEZ

\title{
Construction de flots de Smale en dimension 3
}

Annales de la faculté des sciences de Toulouse $6^{e}$ série, tome $8, \mathrm{n}^{\circ} 3$ (1999), p. 369-410

<http://www.numdam.org/item?id=AFST_1999_6_8_3_369_0>

(C) Université Paul Sabatier, 1999, tous droits réservés.

L'accès aux archives de la revue «Annales de la faculté des sciences de Toulouse » (http://picard.ups-tlse.fr/ annales/) implique l'accord avec les conditions générales d'utilisation (http://www.numdam.org/conditions). Toute utilisation commerciale ou impression systématique est constitutive d'une infraction pénale. Toute copie ou impression de ce fichier doit contenir la présente mention de copyright.

\section{Numdam}

Article numérisé dans le cadre du programme

Numérisation de documents anciens mathématiques

http://www.numdam.org/ 


\title{
Construction de flots de Smale en dimension $3^{(*)}$
}

\author{
F. BÉGuin, C. Bonatti ET J.L. Vieitez ${ }^{(1)}$
}

\begin{abstract}
RÉSUMÉ. - Dans [1], nous montrons que le germe d'un champ de vecteurs $X$, en dimension 3, le long d'un ensemble hyperbolique saturé $K$ détermine de façon unique une variété à bord munie d'un flot (en quelque sorte la variété et le flot les plus simples réalisant le germe considéré) appelés modèle de $(X, K)$. Ce modèle est alors caractérisé à équivalence topologique près par une information combinatoire finie : le type géométrique d'une partition de Markov de $K$. Dans cet article, nous donnons une construction explicite du modèle d'un type géométrique quelconque. Cette construction nous permet de dégager des propriétés des modèles et nous fournit des exemples montrant la différence entre les ensembles hyperboliques des flots en dimension 3 et ceux des suspensions de difféomorphismes de surfaces.
\end{abstract}

Abstract. - The germ of a 3-vector field $X$ along an hyperbolic saturated set $K$ determines uniquely a manifold with boundary carrying a flow (roughly speaking the simpliest mainfold and flow carrying the germ considered above) called the model of $(X, K)$. We have seen in [1] that this model is caracterized up to topological equivalence by a finite combinatorial information : the geometrical type of a Markov partition of $K$. In this article, we give an explicit construction of the model of any geometrical type. From this construction follow properties of models and examples which show the difference existing between hyperbolic sets of 3-flows and hyperbolic sets of suspensions of diffeomorphims of surfaces.

(*) Reçu le 30 mars 1998, accepté le $1^{\text {er }}$ juin 1999

(1) François Béguin, Christian Bonatti, Université de Bourgogne, Laboratoire de Topologie (UMR 5584), BP 400, 21011 Dijon Cedex, France.

José L. Vieitez, Facultad de Ingeniería, C.C. 30, Montevideo, Uruguay.

E-mail: frbeguin@u-bourgogne.fr, bonatti@u-bourgogne.fr, jlvb@fing.edu.uy 


\section{Introduction}

Cet article est une contribution à la classification, à équivalence topologique globale près, des flots de Smale (ou flots $C^{1}$-structurellement stables) des variétés compactes de dimension 3 . Avant de présenter nos propres résultats, rappelons la terminologie et quelques résultats classiques de la théorie hyperbolique de Smale.

\subsection{Rappels sur la théorie de Smale}

Si $X$ et $Y$ sont deux champs de vecteurs de classe $C^{1}$ sur des variétés compactes $\mathcal{M}$ et $\mathcal{N}$, les champs $X$ et $Y$ sont dits topologiquement équivalents s'il existe un homéomorphisme $h$ entre $\mathcal{M}$ et $\mathcal{N}$ envoyant orbite orientée de $X$ sur orbite orientée de $Y$. Les dynamiques globales de $X$ et $Y$ sont alors les mêmes à changement de temps près et au changement de coordonnées $h$ près. Un champ $X$ sur une variété $\mathcal{M}$ est dit $C^{1}$-structurellement stable si, pour tout champ $Y$ sur $\mathcal{M}$, suffisamment $C^{1}$-proche de $X$, les champs $X$ et $Y$ sont topologiquement équivalents ; nous dirons alors plus rapidement que $X$ et son flot sont respectivement un champ et un flot de Smale. Les flots de Smale sont bien sûr les premiers candidats à une classification à équivalence topologique près ; en effet, l'ensemble de leurs classes d'équivalence topologique correspond à un ensemble discret donc dénombrable de flots (l'ensemble des flots $C^{1}$ est métrique séparable).

On rappelle qu'un compact $K$ invariant par le flot d'un champ $X$, disjoint des singularités de $X$, est dit hyperbolique si le fibré tangent à la variété se décompose le long de $K$ en somme directe de trois sous-fibrés continus invariants, $\left.T M\right|_{K}=\mathbb{R} X \oplus E^{s} \oplus E^{u}$, la différentielle du flot (pour un temps fixé suffisamment grand) dilatant uniformément les vecteurs tangents à $E^{u}$ et contractant uniformément ceux tangents à $E^{s}$.

Pour un point $x$ de $K$, on définit alors la variété stable forte $W^{s s}(x)$ comme l'ensemble des points $y$ tels que la distance $d\left(X^{t}(y), X^{t}(x)\right)$ tend vers zéro quand le temps $t$ tend vers $+\infty$ : c'est alors une variété injectivement immergée dans la variété ambiante, et tangente en $x$ à $E^{s}(x)$ (voir [9]). L'orbite par le flot de la variété stable forte de $x$ constitue sa variété stable (faible) notée $W^{s}(x)$. On définit la variété instable de $x$ en remplaçant $X$ par $-X$.

Rappelons également qu'un point est dit errant s'il possède un voisinage $U$ tel que $U$ soit disjoint de $X^{t}(U)$ pour tout $t>1$; on note $\Omega(X)$ l'ensemble des points non-errants. 
C. Robinson et S. Hayashi ont achevé de montrer (voir [8] et [13]) qu'un champ $X$ d'une variété compacte sans bord est $C^{1}$-structurellement stable si et seulement si :

- son ensemble non errant $\Omega(X)$ est constitué d'un nombre fini de singularités hyperboliques et d'un compact hyperbolique sans singularité dans lequel les orbites périodiques sont denses,

- pour tous points $x$ et $y$ de $\Omega(X)$, les variétés invariantes (faibles) $W^{s}(x)$ et $W^{u}(y)$ se coupent transversalement.

La théorie de Smale montre que l'ensemble $\Omega(X)$ d'un flot de Smale se décompose en un nombre fini de compacts transitifs disjoints (dans le cas des flots, ce sont les composantes connexes de $\Omega(X)$ ) : les pièces basiques. Nous voulons classifier non seulement les pièces basiques mais aussi les intersections de leurs variétés invariantes. C'est pourquoi nous allons considérer des ensembles hyperboliques saturés $K$, c'est-à-dire tels que $K=$ $W^{s}(K) \cap W^{u}(K)$, mais non nécessairement transitifs. Pour les flots de Smale en dimension 3, les ensembles hyperboliques saturés sont unions d'un nombre fini de pièces basiques et des intersections de leurs variétés invariantes (voir [4, chapitre 1]). Plus précisément, nous nous intéresserons aux ensembles selles saturés, c'est-à-dire à des ensembles hyperboliques saturés $K$ ne contenant ni attracteur, ni répulseur.

\subsection{But de l'article}

On s'intéresse à la dynamique d'un flot sur des voisinages invariants d'ensembles selles. Dans [1], nous avons montré que le germe $[X, K]$ d'un champ de Smale $X$ le long d'un ensemble selle saturé $K$ ne caractérise (à équivalence topologique près) aucun voisinage invariant. Par contre, un tel germe caractérise une unique variété à bord abstraite, munie d'un champ de vecteurs transverse au bord dont le maximal invariant est de germe $[X, K]$. Plus précisément :

Si $X$ est un champ de vecteur sur une variété $\mathcal{M}$ compacte, orientable, de dimension 3 et si $K$ est un sous-ensemble de $\mathcal{M}$, on note $[X, K]$ le germe de $X$ le long de $K$ : c'est la classe du couple $(X, K)$ pour la relation d'équivalence définie par $(X, K) \sim(Y, L)$ s'il existe des voisinages $U$ et $V$ de $K$ et $L$ tels que les restrictions à $U$ et $V$ des champs $X$ et $Y$ soient topologiquement équivalentes.

DÉFINITION. - Nous appellerons modèle tout couple $(N, Y)$ où $N$ est une variété compacte à bord, orientable, de dimension 3 et où $Y$ est un champ de vecteurs sur $N$, transverse au bord, tels que : 
i) Le maximal invariant de $Y$ dans $N$ est un ensemble selle saturé $K_{Y}$.

ii) Notons $\partial_{1} N$ l'union des composantes connexes du bord de $N$ où $Y$ est rentrant dans $N$; alors tout cercle plongé dans $\partial_{1} N$, disjoint de $W^{s}\left(K_{Y}\right)$ borde un disque dans $\partial_{1} N$ également disjoint de $W^{s}\left(K_{Y}\right)$.

iii) Toute composante connexe de $N$ contient un point de $K_{Y}$.

On dira alors que le couple $(N, Y)$ est un modèle du germe $[X, K]$ si les germes $[X, K]$ et $\left[Y, K_{Y}\right]$ sont les mêmes.

\section{Remarques.}

- Dans la définition ci-dessus, la condition ii) implique une propriété analogue ii') sur l'union $\partial_{2} N$ des composantes connexes du bord de $N$ où $Y$ est sortant de $N$ : tout cercle plongé dans $\partial_{2} N$, disjoint de $W^{u}\left(K_{Y}\right)$ borde un disque dans $\partial_{2} N$ également disjoint de $W^{u}\left(K_{Y}\right)$.

- On a prétendu s'intéresser à des voisinages invariants d'ensembles hyperboliques. Notre définition d'un modèle comme une variété à bords transverses au champ qu'elle porte peu sembler en contradiction avec cet intérêt. Cependant, on peut complèter naturellement tout modèle $(N, Y)$ en une variété ouverte sans bord $\mathcal{N}$ munie d'un champ (que l'on note encore $Y$ ) qui étend $Y$ et telle que $\mathcal{N}$ soit le saturé de $N$ sous l'action de $Y$. La classe d'équivalence topologique de $(N, Y)$ caractérise alors celle de $(\mathcal{N}, Y)$.

Nous avons montré :

ThÉORÈme ([1]). - Pour tout champ de vecteurs $X$ sur une variété compacte orientable $\mathcal{M}$ de dimension 3 et tout ensemble selle saturé $K$ de $X$, il existe un unique modèle du germe $[X, K]$ à équivalence topologique près.

On parlera alors, par abus de langage, du modèle de $[X, K]$ (qui est plutôt une classe d'équivalence topologique qu'un modèle proprement dit). Le modèle de $[X, K]$ est, en quelque sorte, le couple constitué de la variété à bord et du champ de vecteurs les plus simples parmi ceux exhibant le germe $[X, K]$.

Si on considère un difféomorphisme d'une surface compacte, ses compacts hyperboliques saturés admettent de bonnes partitions de Markov par de vrais rectangles plongés. Il en va de même pour les ensembles selles de flots en dimension 3 si on s'intéresse, cette fois, à une section locale de l'ensemble selle considéré et à l'application de premier retour sur cette section. Pour de telles partitions, C. Bonatti et R. Langevin ont introduit 
une combinatoire codant la géométrie des intersections des rectangles de la partition et de leur images : le type géométrique de la partition (voir la sous-partie 1.2). Le type géométrique d'une bonne partition de Markov d'un ensemble selle saturé $K$ caractérise alors le germe de $X$ le long de $K$. Ceci et le théorème précédent montre que deux ensembles selles saturés $K$ et $L$ de flots $X$ et $Y$ admettant des bonnes partitions de Markov de même type géométrique ont des modèles topologiquement équivalents. Ceci nous autorise à parler du modèle d'un type géométrique $T$ : c'est, s'il existe, l'unique modèle (à équivalence topologique près) dont le maximal invariant admet une bonne partition de type géométrique $T$.

Remarquons qu'étant donnée une partition de Markov de type $T$, dans $\mathcal{M}$, d'un ensemble selle $K$, la construction du modèle de $[X, K]$ se fait à partir d'un voisinage de $K$, par des chirurgies qui ne sont pas nécessairement disjointes de la partition de Markov. A priori, le modèle de $[X, K]$ pourrait donc ne contenir aucune partition de Markov de type $T$ de $K$.

Le but de cet article est de donner une construction explicite du modèle d'un type géométrique et d'en tirer des corollaires. Nous allons montrer :

THÉORÈme 0.1. - Pour tout type géométrique abstrait $T$, il existe un modèle dont le maximal invariant admet une bonne partition de Markov de type $T$.

Tout modèle se complète en un champ de Smale non singulier sur une variété compacte de dimension 3 sans bord (voir, par exemple, [6]). Le théorème 0.1 montre donc que tout type géométrique abstrait est le type d'une partition d'un ensemble selle saturé d'un flot de Smale non singulier.

En tant que pur résultat d'existence, le théorème 0.1 est une généralisation d'un théorème de $\mathrm{C}$. Pugh et $\mathrm{M}$. Shub (voir [12]) concernant les suspensions de sous-shifts de types finis. En fait, nous donnons ici une construction du modèle d'un type abstrait quelconque par une suite finie d'opérations élémentaires parmi les suivantes : produit cartésien de segments, quotient par une relation d'équivalence (affine), découpage le long de surfaces constructibles par les opérations précédentes, recollement de variétés le long de parties de leurs bords.

La construction effectuée du modèle d'un type géométrique montre en fait une version plus précise du théorème 0.1 :

Le théorème 0.1 implique que, pour tout ensemble selle saturé $K$ admettant une partition de type $T$, le modèle de $K$ contient une partition de type $T$. Nous aimerions que le plongement de cette partition de Markov 
dans le modèle soit canonique ; c'est-à-dire que nous aimerions que pour deux tels plongements, il existe une équivalence topologique du modèle conjuguant les plongements. Ce n'est pas vrai sans hypothèse supplémentaire sur ces plongements. Cependant, aux rectangles d'une partition de Markov d'un flot en dimension 3 sont naturellement associés des cubes. Une bonne partition de Markov est dite essentielle si l'intersection de toute orbite avec l'union des cubes est connexe (voir la partie 1.1). Nous avons montré que toute équivalence topologique entre les unions des cubes de deux partitions de Markov de même type géométrique se prolonge en une équivalence du modèle (voir [1]). La construction effectuée montre alors :

Corollaire 0.2. - Soit $X$ un champ de vecteurs sur une variété compacte $\mathcal{M}$ de dimension 3 (avec ou sans bord) et soit $K$ un ensemble selle saturé de $X$ admettant, dans $\mathcal{M}$, une bonne partition de Markov de type géométrique $T$. Alors il existe une bonne partition de Markov essentielle de $K$, de type $T$, incluse dans le modèle de $[X, K]$. (Cette partition est donc unique à équivalence topologique globale du modèle près.)

La construction du modèle d'un type géométrique nous permet de construire explicitement des exemples de flots. Nous exhibons ici des champs de Smale en dimension 3 dont le germe le long d'un ensemble selle saturé n'est le germe d'aucune suspension de difféomorphisme de Smale de surface compacte le long d'un ensemble selle saturé (voir la partie 4).

En particulier, le nombre de composantes des bords d'entrée et de sortie du modèle d'un ensemble selle saturé $K$ d'un champ de vecteurs $X$ et le genre de chacune de ces composantes sont bien sûr des invariants associés au germe $[X, K]$. Nous montrons au cours de la construction que ces invariants sont calculables à partir du type géométrique de n'importe quelle partition de Markov de $K$. On montre alors :

Proposition. - Soit $K$ un ensemble selle saturé d'un flot de Smale $X$ sur une variété compacte orientable de dimension 3 dont le germe est le même que celui de la suspension d'un difféomorphisme de Smale de surface compacte le long d'un ensemble selle saturé.

Alors toute composante du bord d'entrée ou de sortie du modèle de $[X, K]$ est de genre inférieur ou égal à 1 (c'est une sphère ou un tore).

Puis, nous construisons un exemple de type géométrique $T$ dont le modèle possède une composante de bord de genre 2 . Cet exemple est très facilement généralisable.

Remarque. - Des travaux antérieurs (voir [3] ou [4]) montrent que certains types géométriques sont irréalisables par des partitions d'ensembles selles saturés de difféomorphismes de Smale de surfaces compactes. Nous 
montrons par un exemple qu'un flot admettant un tel type géométrique non réalisable pourrait cependant être la suspension d'un difféomorphisme (en choisissant judicieusement la transversale). Ceci montre que les travaux de [3] et [4] ne résolvent pas notre problème.

\section{Types géométriques de partitions de Markov}

Le but de cette partie est de définir les notions de bonne partition de Markov, de partition de Markov essentielle et le type géométrique de telles partitions.

\subsection{Bonnes partitions de Markov et partitions de Markov essen- tielles}

Les définitions usuelles de partition de Markov pour les flots dépendent du choix d'une transversale sur laquelle on regarde l'application de premier retour. Nous introduisons la notion de partition de Markov essentielle qui ne dépend pas du choix d'une transversale. On se place dans une variété compacte orientable de dimension 3.

Nous appellerons rectangle $R$ l'image d'un plongement $h$ de $[0,1]^{2}$ dans une surface, $R$ étant muni de deux feuilletages $\mathcal{F}^{s}$ et $\mathcal{F}^{u}$. On demande au feuilletages $\mathcal{F}^{s}$ et $\mathcal{F}^{u}$ d'être de classe $C^{0}$, à feuilles $C^{1}$, triviaux, transverses l'un à l'autre, et tels que $h([0,1] \times\{0\})$ et $h([0,1] \times\{1\})$ soient deux feuilles de $\mathcal{F}^{s}$ et $h(\{0\} \times[0,1])$ et $h(\{1\} \times[0,1])$ soient deux feuilles de $\mathcal{F}^{u}$.

Les feuilles de $\mathcal{F}^{s}$ seront dites horizontales et celles de $\mathcal{F}^{u}$ verticales. Le bord horizontal de $R$ (on dira aussi le bord stable) sera alors $\partial^{s} R=$ $h([0,1] \times\{0\}) \cup h([0,1] \times\{1\})$ et son bord vertical (on dira aussi son bord instable $)$ sera $\partial^{u} R=h(\{0\} \times[0,1]) \cup h(\{1\} \times[0,1])$.

On appellera sous-rectangle horizontal de $R$ tout rectangle $H$ inclus dans $R$ tel que $\partial^{u} H$ soit inclus dans $\partial^{u} R$ et tel que $\partial^{s} H$ soit constitué de deux feuilles du feuilletage $\mathcal{F}^{s}$ de $R$. De même, on appellera sous-rectangle vertical de $R$ tout rectangle $V$ inclus dans $R$ tel que $\partial^{s} V$ soit inclus dans $\partial^{s} R$ et tel que $\partial^{u} H$ soit constitué de deux feuilles du feuilletage $\mathcal{F}^{u}$.

Tout ensemble selle saturé $K$ d'un champ $X$ admet une transversale locale : simplement une surface $\Sigma$ compacte, éventuellement à bords, non nécessairement connexe, transverse à $X$, telle que toute orbite de $K$ coupe $\Sigma$ et telle que le bord de $\Sigma$ soit disjoint de $K$. L'application de premier retour sur cette surface n'est bien sûr que partiellement définie.

Etant donné une transversale locale $\Sigma$ de $K$ et un rectangle $R$ dans $\Sigma$, on dira que le premier retour de $R$ est bien défini si l'orbite positive de tout 
point de $R$ recoupe $\Sigma$ et si le premier retour des points se fait en temps continu.

DÉFINITION. - On appellera bonne partition de Markov de K la donnée d'une transversale locale $\Sigma$ de $K$ et d'une collection finie de rectangles disjoints $R_{1}, \ldots, R_{n}$ dans $\Sigma$ tels que :

- Pour tout $i$, le premier retour de $R_{i}$ sur $\Sigma$ est bien défini; notons le $f\left(R_{i}\right)$,

- $K \cap \Sigma$ est le maximal invariant de l'union des $R_{i}$ pour l'application de premier retour $f$. De plus, il n'y a pas de rectangle inutile : chaque $R_{i}$ contient un point de $K$.

- Pour tous $i$ et $j$, l'intersection $f\left(R_{i}\right) \cap R_{j}$ a un nombre fini de composantes. Chacune de ces composantes est à la fois un sous-rectangle horizontal de $f\left(R_{i}\right)$ et un sous-rectangle vertical de $R_{j}$; de plus, on exige que $\partial^{u} R_{j}$ soit disjoint de cette intersection ainsi que $\partial^{s} f\left(R_{j}\right)$,

- Les feuilletages $\mathcal{F}^{s}$ et $\mathcal{F}^{u}$ sont invariants par $f$ et il existe une métrique sur $\Sigma$ telle que pour tout $x$ dans l'union des $R_{i}$, tel que $f(x)$ soit aussi dans l'union des $R_{i}$, tout vecteur tangent en $x$ à $\mathcal{F}^{s}$ soit strictement contracté par $f$ et tout vecteur tangent en $x$ à $\mathcal{F}^{u}$ soit strictement dilaté par $f$.

\section{Remarques.}

- Les propriétés de contraction et de dilatation exigées dans la définition impliquent que toute feuille de $\mathcal{F}^{s}$ passant par un point de $K$ est un segment de feuille de $W^{s}(K)$ et que toute feuille de $\mathcal{F}^{u}$ passant par un point de $K$ est un segment de feuille de $W^{u}(K)$.

- Chaque composante $C$ de $R \cap f(R)$ contient un point de $K$. En effet, $C$ est alors à la fois un sous-rectangle horizontal de $f\left(R_{i}\right)$ et un sous-rectangle vertical de $R_{j}$ pour un certain couple $(i, j)$. Mais $R_{i}$ contient un point de $K$ par hypothèse et tout sous-rectangle horizontal de $f\left(R_{i}\right)$ est donc traversé par une feuille de $W^{u}(K)$ et, de même, $R_{j}$ contient un point de $K$ par hypothèse et tout sous-rectangle vertical de $R_{j}$ est donc traversé par une feuille de $W^{s}(K)$. Chaque composante de $R \cap f(R)$ contient donc un point de $W^{s}(K) \cap W^{u}(K)=K$.

- Le troisième point de la définition implique que $K \cap \Sigma$ est inclus dans l'intérieur des rectangles $R_{i}$ de la partition. En effet, on a exigé que le bord instable et le l'image par $f$ du bord stable de chaque rectangle soit disjoint du maximal invariant de l'union des $R_{i}$. 
Remarquons qu'a priori le fait qu'une famille de rectangles $\left\{R_{i}\right\}$ forme une bonne partition de Markov dépend de la transversale $\Sigma$ choisie. C'est pourquoi on introduit les définitions suivantes :

A un rectangle $R_{i}$ d'une bonne partition de Markov sur une transversale locale $\Sigma$ est naturellement associé un cube $C_{i}$ union des segments d'orbites joignant un point de $R_{i}$ à son premier retour sur $\Sigma$ (c'est a priori un cube seulement immergé).

DÉfinition. - On dira qu'une bonne partition de Markov de $K$ est essentielle si l'intersection de toute orbite avec l'union des cubes est connexe.

Notons $R$ l'union des rectangles d'une bonne partition $\mathcal{R}=\left\{\Sigma,\left\{R_{i}\right\}\right\}$. La partition $\mathcal{R}$ est essentielle signifie que l'orbite positive de tout point de $f(R) \backslash R$ ne revient jamais couper $R$. Le but de la fin de la sous-partie est de montrer que la notion de partition essentielle ne dépend pas de la transversale $\Sigma$ choisie.

Notons $P$ l'application de premier retour partiellement définie de $R$ sur $R$. Rappelons qu'on note $f$ l'application de premier retour (définie en tout point) de $R$ sur $\Sigma$. Bien sûr $f^{-1}(R) \cap R$ est inclus dans le domaine de définition $\operatorname{Dom}(P)$ de $P$.

Remarque. - La partition $\mathcal{R}=\left\{\Sigma,\left\{R_{i}\right\}\right\}$ est essentielle si et seulement si le domaine de définition $\operatorname{Dom}(P)$ est égal à $f^{-1}(R) \cap R$. En effet, la partition est essentielle si et seulement si il n'existe pas d'orbite dont l'intersection avec les cubes est disconnexe ; c'est-à-dire s'il n'existe aucun segment d'orbite d'intérieur disjoint des cubes, à extrémités sur la face de sortie $f(R) \backslash R$ des cubes et sur la face d'entrée contenue dans $R$ des cubes. Autrement dit, la partition est essentielle si et seulement si il n'existe aucun point $x \in R$ tel que $f(x) \notin R$ mais tel que $P$ soit défini en $x$.

On peut maintenant montrer :

Proposition 1.1. - Soit $\left\{\Sigma,\left\{R_{i}\right\}\right\}$ une bonne partition de Markov de $K$ essentielle et soit $\tilde{\Sigma}$ une autre transversale contenant les $R_{i}$ tel que $\left\{\tilde{\Sigma},\left\{R_{i}\right\}\right\}$ soit aussi une bonne partition de Markov de K. Alors $\left\{\tilde{\Sigma},\left\{R_{i}\right\}\right\}$ est une bonne partition de Markov essentielle de $K$.

Démonstration. - On note $R$ l'union des rectangles $R_{i}$. On note $f, \tilde{f}$ et $P$ les applications de premier retour de $R$ sur $\Sigma, \tilde{\Sigma}$ et $R$ respectivement.

On a vu que $\tilde{f}^{-1}(R) \cap R$ est inclus dans le domaine $\operatorname{Dom}(P)$ de $P$. D'après la remarque ci-dessus, nous savons que $f^{-1}(R) \cap R$ est égal à $\operatorname{Dom}(P)$ et nous devons montrer que $\tilde{f}^{-1}(R) \cap R$ est égal à $\operatorname{Dom}(P)$. 
On commence par remarquer que chaque composante $C$ de $\operatorname{Dom}(P)$ contient un point $x$ de $K$. En effet, $\operatorname{Dom}(P)$ est égal à $f^{-1}(R) \cap R$ et la remarque suivant la définition de bonne partition de Markov conclut. Puisque $K \cap \tilde{\Sigma}$ est le maximal invariant de $R$ sous l'action de $\tilde{f}$, le point $x$ appartient nécessairement à $\tilde{f}^{-1}(R) \cap R$.

On s'intéresse maintenant à la composante connexe $\tilde{C}$ de $\tilde{f}^{-1}\left(R_{i}\right) \cap R_{j}$ contenant $x$. On a bien sûr $\tilde{C} \subset C$ et on veut montrer l'égalité. Par définition d'une partition de Markov, les composantes $C$ et $\tilde{C}$ sont toutes deux des sous-rectangles horizontaux de $R_{j}$. Pour montrer l'égalité des composantes $C$ et $\tilde{C}$, il suffit donc de montrer que le bord stable de $\tilde{C}$ est inclus dans celui de $C$. Cependant, $\tilde{f}^{-1}$ coïncide avec $P^{-1}$ sur $R \cap \tilde{f}(R)$ et donc, a fortior $i$ $\operatorname{sur}\left(\partial^{s} R_{i} \cap \tilde{f}\left(R_{j}\right)\right) \subset\left(\partial^{s} R_{i} \cap P\left(R_{j}\right)\right)$. Le bord stable $\tilde{f}^{-1}\left(\partial^{s} R_{i}\right) \cap R_{j}$ de $\tilde{C}$ est donc inclus dans le bord stable $P^{-1}\left(\partial^{s} R_{i}\right) \cap R_{j}$ de $C$ ce qui conclut.

On a donc montré que toute $C$ de $\operatorname{Dom}(P)$ est incluse dans $\tilde{f}^{-1}(R) \cap R$ ce qui conclut la preuve.

Un argument similaire montre la proposition suivante qui nous permet de donner une définition intrinsèque (sans référence à une transversale) d'une partition essentielle :

Proposition 1.2. - Une famille de rectangles disjoints $\left\{R_{i}\right\}$ transverses au flot admet une transversale $\Sigma$ qui en fait une partition essentielle de $K$ si et seulement si :

- l'application de premier retour $P$ de $R=\cup_{i} R_{i}$ sur $R$ est définie sur un nombre fini de sous-rectangles horizontaux disjoints et disjoints du bord horizontal des $R_{i}$ vers une union finie de sous-rectangles verticaux disjoints et disjoints des bords verticaux des $R_{i}$.

\section{- le maximal invariant de $P$ est égal à $K \cap R$}

- $P$ ainsi que le temps de retour sur $R$ est continue et différentiable

$-P$ laisse invariants les feuilletages horizontaux et verticaux des $R_{i}$ et possède les propriétés de contraction et dilatation de ces feuilletages.

Rappelons que nous avons montré dans [1, proposition 2.8] que tout ensemble selle saturé possède une partition de Markov essentielle. 


\subsection{Types géométriques abstraits et type géométrique d'une par- tition de Markov}

On ne veut retenir d'une partition de Markov qu'une information combinatoire finie: la façon dont chaque $f\left(R_{i}\right)$ coupe les différents $R_{j}$, c'est-à-dire selon combien de sous-rectangles verticaux, lesquels, dans quel ordre et dans quels sens:

DÉFINITION. - On appellera type géométrique abstrait la donnée de :

- Un entier $n \in \mathbb{N} \backslash\{0\}$ $\sum_{i} v_{i}$

- Pour tout $i \in\{1, \ldots, n\}$ deux entiers $h_{i}, v_{i} \in \mathbb{N} \backslash\{0\}$ tels que $\sum_{i} h_{i}=$

- Une application $\Phi$ de l'ensemble $\left\{(i, j) / i \in\{1, \ldots, n\}, j \in\left\{1 \ldots, h_{i}\right\}\right\}$ dans l'ensemble $\left\{(k, l) / k \in\{1, \ldots, n\}, l \in\left\{1, \ldots, v_{k}\right\}\right\} \times\{-,+\}$, induisant une bijection par "oubli des signes"

A une bonne partition de Markov dans une variété orientable, on peut associer un type géométrique. On procède comme suit.

La variété étant orientée, tout rectangle transverse au flot hérite d'une orientation. Le choix d'une orientation des horizontales d'un rectangle induit donc une orientation des verticales. Soit $\mathcal{R}=\left\{\Sigma,\left\{R_{i}\right\}\right\}$ une bonne partition de Markov et un choix des orientations des horizontales et des verticales des rectangles de $\mathcal{R}$. Le type géométrique de $\mathcal{R}$ est un type géométrique abstrait comme défini ci-dessus tel que :

- $n$ est le nombre de rectangles de $\mathcal{R}$,

- Notons $f\left(R_{i}\right)$ le premier retour de $R_{i}$ sur $\Sigma$. Pour tout $i \leqslant n, h_{i}$ est le nombre de composantes connexes de l'intersection de $f\left(R_{i}\right)$ avec l'union des $R_{j}$. Ces composantes connexes sont, par définition d'une bonne partition de Markov, les images de sous-rectangles horizontaux de $R_{i}$. On notera $H_{i}^{1} \ldots H_{i}^{h_{i}}$ ces sous-rectangles numérotés dans l'ordre induit par l'orientation des verticales de $R_{i}$.

- De même, pour tout $k \leqslant n, v_{k}$ est le nombre de composantes connexes de l'intersection de $R_{k}$ avec l'union des $f\left(R_{j}\right)$. On notera $V_{k}^{1} \ldots V_{k}^{v_{k}}$ ces sousrectangles verticaux de $R_{k}$ numérotés dans l'ordre induit par l'orientation des horizontales de ce rectangle.

- Chaque sous-rectangle horizontal $H_{i}^{j}$ de $R_{i}$ est envoyé par $f$ sur un sous-rectangle vertical $V_{k}^{l}$ de $R_{k}$. L'application $\Phi$ envoie alors $(i, j)$ sur 
$((k, l), \varepsilon)$ où $\varepsilon=+1$ si l'orientation des verticales de $f\left(H_{i}^{j}\right)$ coïncide avec celle des verticales de $V_{k}^{l}$ et $\varepsilon=-1$ sinon. (voir la figure 1 ).

Si $\mathcal{R}$ est essentielle, son type géométrique ne dépend bien sûr pas de la transversale $\Sigma$.

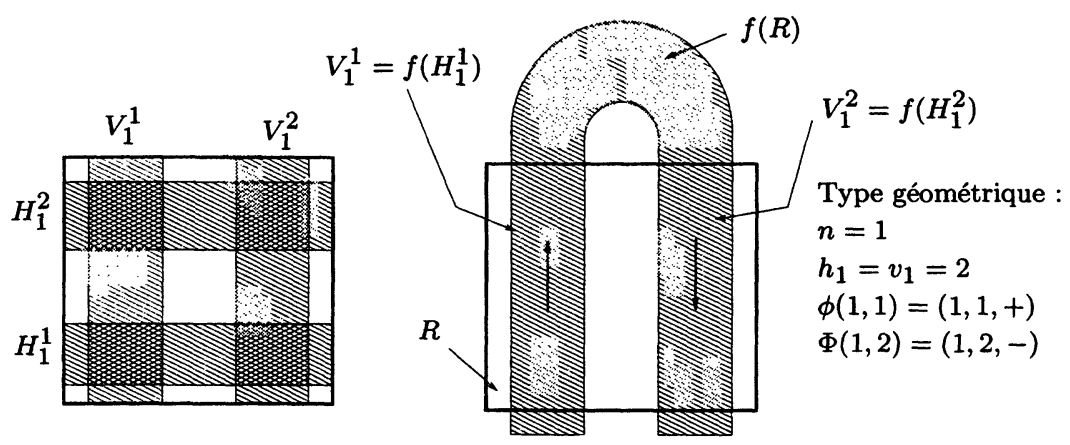

Fig. 1 Le type géométriqúe du fer à cheval de Smale

Remarque. - Dans le cas où $K$ est transitif, il nous semble que l'information donnée par le type géométrique d'une partition de Markov de $K$ est la même que celle donnée par un gabarit (template en anglais) non plongé mais dont les feuillets arrivant au lieu singulier (joining chart) sont ordonnés. Voir l'introduction de la notion de template dans [2] ou encore [7].

\section{Patrons et ouvrages}

On se fixe maintenant et pour toute la suite un type géométrique abstrait $T$ quelconque. La donnée du type $T$ est la donnée de $\left(n,\left\{h_{i}\right\},\left\{v_{i}\right\}, \Phi\right)$, où $\Phi$ est une application de $\left\{i \in\{1, \cdots, n\}, j \in\left\{1, \cdots, h_{i}\right\}\right\}$ dans $\{k \in$ $\left.\{1, \cdots, n\}, l \in\left\{1, \cdots, v_{k}\right\}\right\} \times\{-,+\}$.

On cherche à reconstruire une dynamique admettant une partition de type $T$. On commence par construire un difféomorphisme partiellement défini sur des rectangles en suivant la règle donnée par $T$. On obtient ainsi un patron de $T$ (c'est une légère modification de la notion de réalisation de [4] et [10]). Le but étant la construction d'un représentant de la classe d'équivalence du modèle d'un type géométrique, on travaillera avec les objets les plus simples possibles : les patrons considérés seront affines.

DÉFINITION. - Un patron (affine) $P$ du type géométrique $T$ sera la donnée de : 
$-n$ rectangles orientés affines de $\mathbb{R}^{2}$ notés $R_{i}$ avec des feuilletages horizontaux et verticaux affines, une orientation des horizontales (les verticales sont alors orientées pour former une base directe).

- pour tout $i$ compris entre 1 et $n$, de $h_{i}$ sous-rectangles horizontaux $H_{i}^{j}$ de $R_{i}$ deux à deux disjoints, disjoints de $\partial^{s} R_{i}$ et numérotés en suivant l'orientation des verticales de $R_{i}$,

- pour tout $i$ compris entre 1 et $n$, de $v_{i}$ sous-rectangles verticaux $V_{i}^{j}$ de $R_{i}$ deux à deux disjoints, disjoints de $\partial^{u} R_{i}$ et ordonnés suivant l'orientation des horizontales,

- un difféomorphisme $\varphi$ de l'union $\bigcup_{i, j} H_{i}^{j}$ dans l'union $\bigcup_{k, l} V_{k}^{l}$ vérifiant : pour tous $i, j, k, l$ tels que $\Phi(i, j)=((k, l), \varepsilon)$ avec $\varepsilon= \pm 1$, la restriction de $\varphi \grave{a} H_{i}^{j}$ est un difféomorphisme affine dans $V_{k}^{l}$ préservant l'orientation, laissant invariants les feuilletages horizontaux et les feuilletages verticaux des rectangles $R_{i}$, préservant ou inversant l'orientation des verticales suivant que $\varepsilon$ vaut 1 ou -1 , contractant strictement la direction horizontale et dilatant strictement la direction verticale.

Lemme 2.1. - Pour tout type géométrique $T$, il existe un patron de $T$.

Démonstration. - Le seul point éventuellement non évident est l'obtention des propriétés de dilatation-contraction. Les sous-rectangles horizontaux et verticaux étant disjoints des bords verticaux et horizontaux des $R_{i}$, ils sont respectivement de hauteur et de largeur inférieures à celles des $R_{i}$ les contenant. Il suffit de choisir les rectangles $R_{i}$ tous de même hauteur et de même largeur pour obtenir ces propriétés.

Soit $P=\left\{R_{i}, H_{i}^{j}, V_{k}^{l}, \varphi\right\}$ un patron de $T$. On notera $R$ l'union des rectangles $R_{i}$, et de même, $H=\cup_{i, j} H_{i}^{j}$ et $V=\cup_{k, l} V_{k}^{l}$. Comme l'application $\varphi$ du patron n'est définie que sur un sous-ensemble des $R_{i}$, nous donnons un sens à sa suspension : on définit des espaces topologiques $\mathcal{P}_{p, q}$ qui seront les unions de segments d'orbites de points des rectangles du patron entre les temps $p$ et $q$.

DÉfINITION. - Pour tous réels $p \leqslant q$, on appellera ouvrage $\mathcal{P}_{p, q}$ associé au patron $P$ l'ensemble :

$$
\mathcal{P}_{p, q}=(R \times[p, q]) / \sim
$$

où $\sim$ est la relation d'équivalence engendrée par $(x, t) \sim\left(\varphi^{-1}(x), t+1\right)$. On notera plus rapidement $\mathcal{P}_{\infty}=\mathcal{P}_{-\infty,+\infty}=(R \times \mathbb{R}) / \sim$. 


\section{Remarques.}

- Deux points $(x, t)$ et $(y, s)$ de $R \times[p, q]$ sont équivalents par $\sim \mathrm{si}$ $s-t=n \in \mathbb{Z}$, et si $\varphi^{n}(y)$ est défini et égal à $x$. On en déduit que la relation $\sim$ est fermée dans $R \times[p, q]$ (le domaine de définition de $\varphi$ est fermé) et donc que les $\mathcal{P}_{p, q}$ sont des espaces topologiques séparés (pour $p$ et $q$ finis).

- Soit $p^{\prime} \leqslant p \leqslant q \leqslant q^{\prime}$ (éventuellement infinis). Si $(x, t)$ et $(y, s)$ sont dans $R \times[p, q]$ et sont équivalents dans $\mathcal{P}_{p^{\prime}, q^{\prime}}$ alors ils sont aussi équivalents dans $\mathcal{P}_{p, q}$. On a donc un plongement naturel de $\mathcal{P}_{p, q}$ dans $\mathcal{P}_{p^{\prime}, q^{\prime}}$. On notera par la suite $\pi$ la projection de chaque $R \times \mathbb{R}$ sur $\mathcal{P}_{\infty}$. L'image par $\pi$ de $R \times[p, q]$ est alors $\mathcal{P}_{p, q}$.

- L'ensemble $\mathcal{P}_{\infty} \cap\{t \in \mathbb{Z}\}$ est bien défini (si $(x, t)$ et $(y, s)$ appartenant à $R \times \mathbb{R}$ sont identifiés alors $s-t=n \in \mathbb{Z}$ ) ce qui permet d'effectuer des schémas de $\mathcal{P}_{p, q}$ en coupe par $\{t \in \mathbb{Z}\}$.
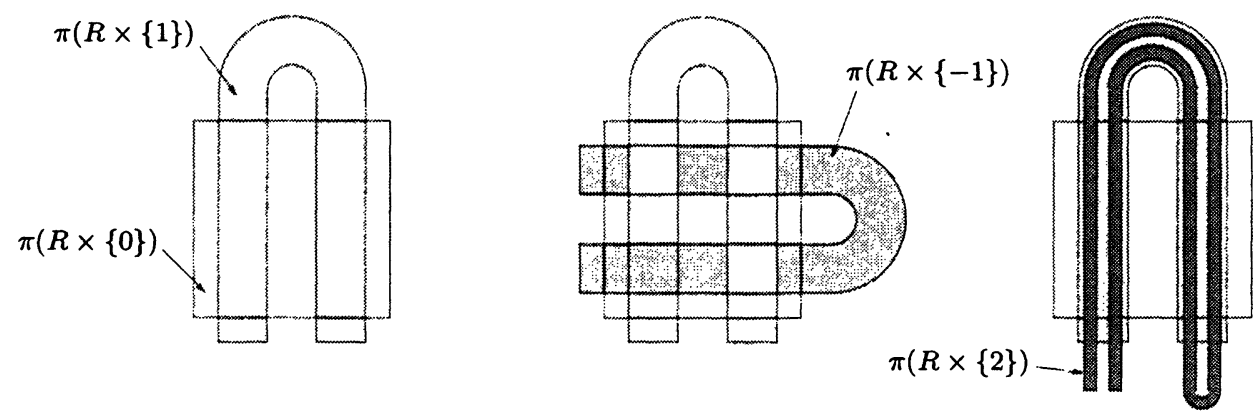

Fig. 2 Vues de $\mathcal{P}_{0,1}, \mathcal{P}_{-1,1}$, et $\mathcal{P}_{0,2}$ (intersectés par $\{t \in \mathbb{Z}\}$ ) pour un patron du type géométrique à un rectangle du fer à cheval usuel

A priori, $\varphi$ n'étant pas définie sur les $R_{i}$ en entier, les ouvrages $\mathcal{P}_{p, q}$ sont des variétés branchées. On va montrer qu'en fait, à cause de la nature du lieu de recollement par $\sim$, les ouvrages sont des variétés (non branchées) compactes à bord arêtes et coins.

Proposition 2.2 Pour tous réels $p, q \in \mathbb{R}$ (finis), $\mathcal{P}_{p, q}$ est une variété différentielle (affine) séparée, compacte, de dimension 3 , à bord, arêtes et coins.

Démonstration. - Dans le cas où $q-p>1$, nous allons montrer qu'il existe un atlas $\mathcal{A}=\left(U_{i}, \psi_{i}\right)$ de $\mathcal{P}_{p, q}$ dont les cartes sont des $\psi_{i}: U_{i} \rightarrow V_{i}$ où $V_{i}$ est un voisinage de l'origine dans l'un des sous-ensembles de $\mathbb{R}^{3}$ suivants : 
1) $\mathbb{R}^{3}$ (point intérieur)

2) $\mathbb{R}^{+} \times \mathbb{R}^{2}$ (point sur une face)

3) $\left(\mathbb{R}^{+}\right)^{2} \times \mathbb{R}$ (point sur une arête convexe)

4) $\overline{\mathbb{R}^{3} \backslash\left(\left(\mathbb{R}^{+}\right)^{2} \times \mathbb{R}\right)}$ (point sur une arête concave)

5) $\left(\mathbb{R}^{+}\right)^{3}$ (coin convexe)

6) $\overline{\mathbb{R}^{3} \backslash\left(\left(\mathbb{R}^{+} \times \mathbb{R}^{-} \times \mathbb{R}\right) \cup\left(\mathbb{R}^{+} \times \mathbb{R} \times \mathbb{R}^{-}\right)\right)}$(coin "semiconcave")

et où $\psi_{i} \circ \pi$ envoie, un à un, les trois feuilletages unidimensionnels standards de $R \times[p, q]$ sur les trois feuilletages unidimensionnels standards des $V_{i}$. Les changements de cartes seront chacun des restrictions d'éléments de $(\operatorname{Aff}(\mathbb{R}))^{3}$ (où $\operatorname{Aff}(\mathbb{R})$ désigne le groupe des transformations affines de $\mathbb{R}$ ).

Le cas où $q-p=1$ est beaucoup plus simple, mais introduit pourtant un type de coin de plus. Comme nous n'aurons pas besoin de ce cas particulier, nous l'évincerons de la démonstration.

Nous devons donc comprendre la structure d'un voisinage de tout point de $\mathcal{P}_{p, q}$. Tout point de $\mathcal{P}_{p, q}$ est une classe d'équivalence pour $\sim$. Un voisinage d'un point est obtenu en recollant par $\sim$ un voisinage dans $R \times[p, q]$ de chaque point de la classe.

Toute classe d'équivalence de $\mathcal{P}_{p, q}$ est de la forme $(x, t),\left(\varphi^{-1}(x), t+\right.$ 1), $\ldots,\left(\varphi^{-k}(x), t+k\right)$ avec $0 \leqslant k \leqslant q-p$ (l'entier $k$ dépendant bien sûr du point $(x, t))$. De plus, pour tout $i$ différent de 0 et $k$, le point $\varphi^{-i}(x)$ est dans l'intérieur de $R$. En effet, ses images $\operatorname{par} \varphi$ et $\varphi^{-1}$ sont définies. Il est donc dans l'intersection d'un sous-rectangle horizontal et d'un sous rectangle vertical et, par conséquent, dans l'intérieur des $R_{i}$.

On se fixe maintenant un couple $(x, t)$ de $R \times[p, q]$ "premier dans sa classe" (c'est-à-dire un couple $(x, t)$ dont la classe s'écrit $(x, t), \ldots,\left(\varphi^{-k}(x), t+\right.$ $k)$ ). Nous avons trois cas à considérer suivant que $k$ est égal à 0,1 ou supérieur à 2 . Le cas $k=0$ est très simple, le cas $k=1$ est $a$ priori facile car il suffit de recoller deux ouverts ; la difficulté provient pourtant de faire la liste de tous les cas d'ouverts à recoller possibles, nous le garderons pour la fin.

\section{Cas où $k=0$}

Suivant que $x$ est dans l'intérieur, sur un bord ou égal à un coin d'un $R_{i}$ et que $t$ est égal à $p, q$ ou appartient à $] p, q[$, on a une carte de type 1), 2), 
$3), 5)$. En effet, un voisinage de $(x, t)$ dans $R_{i} \times[p, q]$ est un voisinage dans $\mathcal{P}_{p, q}$.

Cas où $k \geqslant 2$

Remarque. - Soit $y$ un point de $R$ tel que $\varphi^{-2}(y)$ soit défini. Alors il existe un voisinage de $y$ dans $R$ sur lequel $\varphi^{-1}$ est partout définie.

En effet, $\varphi^{-1}$ est défini en un point $z$ si et seulement si $z$ est dans $V=\cup_{k, l} V_{k}^{l}$. Si $\varphi^{-2}(y)$ est défini, alors $y$ n'est pas dans un côté vertical d'un $V_{k}^{l}$ : en effet, l'image par $\varphi^{-1}$ des côtés verticaux d'un $V_{k}^{l}$ est incluse dans les côtés verticaux des $R_{i}$ et est donc disjointe du domaine de définition de $\varphi^{-1}$. Il existe alors un voisinage de $y$ dans $R$ qui est inclus dans $V$, ce qui conclut.

De même, si $y$ est un point de $R$ tel que $\varphi^{2}(y)$ est défini, alors il existe un voisinage de $y$ dans $R$ sur lequel $\varphi$ est partout définie.

LEMME 2.3. - Si $k \geqslant 2$, la classe de $(x, t)$ admet un voisinage muni d'une carte de type 1).

Démonstration. - Pour tout $j$ dans $\{0 \cdots(k-2)\}$, on a, d'après la remarque ci-dessus, tout un voisinage de $y=\varphi^{-j}(x)$ dans $R$ où $\varphi^{-1}$ est définie. De même, pour tout indice $j$ dans $\{2 \cdots k\}$, il existe un voisinage de $y=\varphi^{-j}(x)$ où $\varphi$ est défini.

On en déduit que, pour tout $j$ dans $\{1 \cdots(k-2)\}, \varphi^{-j}(x)$ a un voisinage dans $R$ qui est envoyé difféomorphiquement par $\varphi^{-1}$ sur un voisinage de $\varphi^{-(j+1)}(x)$ dans $R$. De plus ce voisinage est un disque $D_{j}$ centré en $\varphi^{-j}(x)$ puisque $\varphi^{-j}(x)$ est dans l'intérieur des rectangles.

Les points $\left(\varphi^{-1}(x), t+1\right), \ldots,\left(\varphi^{-(k-1)}(x), t+(k-1)\right)$ possèdent donc des voisinages $\left.U_{j}=D_{j} \times\right] t+j-\varepsilon, t+j+\varepsilon[$ dans $R \times[p, q]$ qui sont identifiés points à points dans $\mathcal{P}_{p, q}($ en effet $(t+1), \ldots, t+(k-1)$ sont dans l'intérieur de $[p, q])$.

Si $D_{j}$ est pris assez petit et si $\varepsilon$ est pris également assez petit (en fait strictement plus petit que $1 / 2$ ), il n'y a aucune identification de deux points d'un même $U_{j}$. Dans ce cas, $U_{j}$ se plonge dans $\mathcal{P}_{p, q}$. Il suffit donc de voir qu'il se plonge en un voisinage de la classe de $(x, t)$ pour avoir une carte de type 1).

Pour montrer que $U_{1}$ (ou $U_{2}, \ldots$, ou $U_{k-1}$ ) induit un voisinage de la classe de $(x, t)$ dans $\mathcal{P}_{p, q}$, il reste donc à voir qu'un voisinage de $(x, t)$ dans $R \times \mathbb{R}$ 
est identifié par $\sim$ à un sous-ensemble de $U_{1}$ et, de même, que tout un voisinage de $\left(\varphi^{-k}(x), t+k\right)$ est identifié à tout ou partie de $U_{1}$. C'est bien le cas puisque $\varphi^{-1}$ est défini au voisinage de $x$ (voir la remarque) donc tout point d'un voisinage de $(x, t)$ dans $R \times \mathbb{R}$ est identifié à un point au voisinage de $\left(\varphi^{-1}(x), t+1\right)$ (mais pas réciproquement). De même, $\varphi$ est défini au voisinage de $\varphi^{-k}(x)$ donc tout point d'un voisinage de $\left(\varphi^{-k}(x), t+k\right)$ est identifié à un point au voisinage de $\left(\varphi^{-(k-1)}(x), t+(k-1)\right)$ donc à un point au voisinage de $\left(\varphi^{-1}(x), t+1\right)$.

Cas où $k=1$

La classe de $(x, t)$ ne contient maintenant que deux éléments de $R \times[p, q]$, les points $(x, t)$ et $\left(\varphi^{-1}(x), t+1\right)$.

Remarque. - $\mathrm{Si}(x, t)$ possède un voisinage dans $R \times[p, q]$ qui est identifié à une partie d'un voisinage de $\left(\varphi^{-1}(x), t+1\right)$ dans $R \times[p, q]$, alors un voisinage de la classe de $(x, t)$ dans $\mathcal{P}_{p, q}$ est difféomorphe à un voisinage de $\left(\varphi^{-1}(x), t+1\right)$ dans $R \times[p, q]$. On a alors une carte de type 1$\left.\left.), 2\right), 3\right)$ ou 5$)$. De même, si les rôles de $(x, t)$ et $\left(\varphi^{-1}(x), t+1\right)$ sont inversés.

La remarque traite les cas :

- où $\varphi^{-1}$ est défini au voisinage de $x$ et $t \neq q-1$

- où $\varphi$ est défini au voisinage de $\varphi^{-1}(x)$ et $t \neq p$

Par contraposée, il nous reste cinq cas :

a) $\varphi^{-1}$ n'est pas défini au voisinage de $x, \varphi$ n'est pas défini au voisinage de $\varphi^{-1}(x)$ et $t$ est différent $p$ et de $q-1$.

b) $\varphi^{-1}$ n'est pas défini au voisinage de $x, \varphi$ est défini au voisinage de $\varphi^{-1}(x)$ et $t=p$

c) $\varphi^{-1}$ est défini au voisinage de $x, \varphi$ n'est pas défini au voisinage de $\varphi^{-1}(x)$ et $t=q-1$

d) $\varphi^{-1}$ n'est pas défini au voisinage de $x, \varphi$ n'est pas défini au voisinage de $\varphi^{-1}(x)$ et $t=p$

e) $\varphi^{-1}$ n'est pas défini au voisinage de $x, \varphi$ n'est pas défini au voisinage de $\varphi^{-1}(x)$ et $t=q-1$

Comme $\{t=q-1$ et $t=p\}$ est exclu par hypothèse $(q-p>1)$, la liste des cas est complète. D'autre part, les cas b) et c) sont symétriques ainsi que les cas d) et e). On traitera les cas a), b) et d). 
Remarque. - Dans les cas considérés, $\varphi^{-1}$ est défini en $x$ donc $x$ est dans un sous-rectangle vertical $V_{k}^{l}$. Alors, on rappelle que $\varphi^{-1}$ n'est pas définie sur un voisinage de $x$ dans $R_{k}$ si et seulement si $x$ est dans le bord vertical de $V_{k}^{l}$ ou, de façon équivalente, si et seulement si $\varphi^{-1}(x)$ est dans le bord vertical d'un $R_{i}$.

De même, $\varphi$ est défini en $\varphi^{-1}(x)$ donc $\varphi^{-1}(x)$ est dans un sous-rectangle horizontal $H_{i}^{j}$. Alors $\varphi$ n'est pas défini sur un voisinage de $\varphi^{-1}(x)$ dans $R_{i}$ si et seulement si $\varphi^{-1}(x)$ est dans le bord horizontal de $H_{i}^{j}$ ou, de façon équivalente, si et seulement si $x$ est dans le bord horizontal de $R_{k}$.

Dans le cas a), un voisinage de $(x, t)$ dans $R \times[p, q]$ est donc un demi espace limité par un plan horizontal tangent au champ, de même, un voisinage de $\left(\varphi^{-1}(x), t+1\right)$ est un demi-espace limité par un plan vertical tangent au champ; on vérifie qu'en recollant ces deux voisinages, on obtient une carte de type 4) (arête concave).

De même, dans les cas b) et c), on obtient une carte de type 4) (arête concave) (on recolle ici deux demi-espaces, l'un tangent, l'autre transverse au champ).

Enfin, dans les cas d), un voisinage de $(x, t)$ dans $R \times[p, q]$ est donc un quart d'espace limité par un plan horizontal tangent au champ et un plan transverse au champ, et un voisinage de $\left(\varphi^{-1}(x), t+1\right)$ est un demi-espace limité par un plan vertical tangent au champ; on vérifie qu'en recollant ces deux voisinages, on obtient une carte de type 6) (coin semiconcave).

De même, dans le cas e), on obtient une carte de type 6).

Corollaire 2.4. - Tout point $\pi(x, t)$ de $\mathcal{P}_{\infty}$ possède un voisinage inclus dans $\mathcal{P}_{t-3, t+3}$ (via le plongement naturel de $\mathcal{P}_{t-3, t+3}$ dans $\mathcal{P}_{\infty}$ ). Par conséquent, $\mathcal{P}_{\infty}$ est une variété séparée à bords, arêtes et coins.

Démonstration. - Soit $(x, t)$ un point de $R \times \mathbb{R}$. Notons, comme dans la démonstration ci-dessus, $k_{p, q}$ le nombre d'éléments distincts de $(x, t)$ dans sa classe d'équivalence dans $R \times[p, q]$.

- Si $k_{t-3, t+3}$ est nul alors il existe un voisinage $U$ de $x$ dans $R$ sur lesquels $\varphi$ et $\varphi^{-1}$ ne sont définis nulle part. Alors l'ensemble $\left.U \times\right] t-\varepsilon, t+\varepsilon[$ $\left(\varepsilon<\frac{1}{2}\right)$ est un ouvert de $R \times \mathbb{R}$, saturé pour $\sim$. Sa projection est donc un voisinage ouvert de $\pi(x, t)$ dans $\mathcal{P}_{\infty}$.

- Si $k_{t-3, t+3}$ est supérieur ou égal à 2 , alors, on a vu que $x, \varphi(x)$ ou $\varphi^{-1}(x)$ est dans l'intérieur d'un des $R_{i}$. Le cube ouvert $\mathcal{O}=\operatorname{int}\left(R_{i} \times\right] t-$ $1 / 4, t+1 / 4[)$ est plongé dans $\mathcal{P}_{p, q}$ pour tous $p<t-1 / 4$ et $q>t+1 / 4$ et $\mathcal{P}_{p, q}$ étant une variété, $\mathcal{O}$ est un ouvert dans $\mathcal{P}_{p, q}$. Le saturé pour $\sim$ de $\mathcal{O}$ 


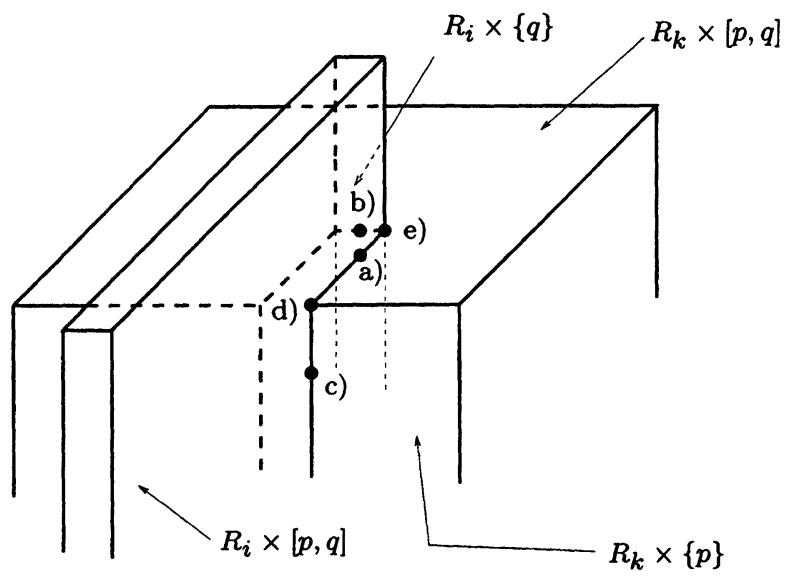

Fig. 3 Différents types de positions dans $\mathcal{P}_{p, q}$

est ouvert dans $R \times[p, q]$ pour tous $p$ et $q$. Le saturé de $\mathcal{O}$ par $\sim$ est donc ouvert dans $R \times \mathbb{R}$. Le plongement de $\mathcal{O}$ dans $\mathcal{P}_{\infty}$ est un voisinage ouvert de $\pi(x, t)$ dans $\mathcal{P}_{\infty}$.

- Si $k_{t-3, t+3}$ est égal à 1 alors $\varphi^{2}(x)$ et $\varphi^{-2}(x)$ ne sont pas définis et $\varphi(x)$ ou $\varphi^{-1}(x)$ n'est pas défini. Pour fixer les idées, supposons que $\varphi^{-1}(x)$ n'est pas défini et que $\varphi(x)$ est donc défini. Il existe un voisinage $U_{x}$ de $x$ dans $R$ où $\varphi^{-1}$ et $\varphi^{2}$ ne sont pas définis et il existe un voisinage $U_{\varphi(x)}$ où $\varphi$ et $\varphi^{-2}$ ne sont pas définis. Alors, pour $\varepsilon<\frac{1}{2}$, l'union de $\left(U_{x} \times\right] t-\varepsilon, t+\varepsilon[)$, de $\left(\varphi\left(U_{x}\right) \times\right] t-1-\varepsilon, t-1+\varepsilon[)$, de $\left(U_{\varphi(x)} \times\right] t-1-\varepsilon, t-1+\varepsilon[)$ et de $\left(\varphi^{-1}\left(U_{\varphi(x)}\right) \times\right] t-\varepsilon, t+\varepsilon[)$ est un ensemble saturé pour $\sim$ dans $R_{i} \times \mathbb{R}$. D'autre part, pour tout $p \leqslant t-3$ et $q \geqslant t+3$, on a vu que cet ensemble induit un voisinage dans $\mathcal{P}_{p, q}$. Il contient donc un ouvert saturé dans $R \times[p, q]$ qui est disjoint de $R \times]-\infty, p]$ et $R \times[q,+\infty[$ et donc ouvert dans $R \times \mathbb{R}$.

On a donc montré que tout point $(x, t)$ de $\mathcal{P}_{\infty}$ possède un voisinage contenu $\mathcal{P}_{t-3, t+3}$, ce qui conclut.

\section{Remarques.}

- Le champ de vecteur $\frac{\partial}{\partial t}$ de $R \times \mathbb{R}$ se projette naturellement sur un champ de vecteurs $X$ de $\mathcal{P}_{\infty}$. L'image de tout point $\pi(x, u)$ de $\mathcal{P}_{\infty}$ par le temps $t$ du flot de $X$ est $\pi(x, u+t)$. On remarque que le champ $X$ est tangent au bord de $\mathcal{P}_{\infty}$ et que son flot $X^{t}$ envoie $\mathcal{P}_{p, q}$ sur $\mathcal{P}_{p+t, q+t}$. 


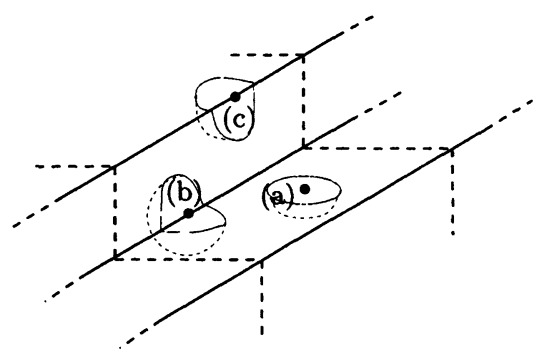

Fig. 4 Points ayant différents types de voisinages dans $\mathcal{P}_{\infty}$

- Tous les points de $\mathcal{P}_{\infty}$ possèdent un voisinage qui est soit une boule (point intérieur), soit une demi-boule (point sur le bord de $\mathcal{P}_{\infty}$ mais pas sur une arête, point (a) sur la figure 4), soit un quart de boule (point (b) sur une arête convexe), soit enfin trois quarts de boule (point (c) sur une arête concave). Le champ $X$ est tangent non seulement au bord, mais aussi aux arêtes.

La fin de cette partie est destinée à montrer que $X$ admet un ensemble selle saturé (le maximal invariant de $\mathcal{P}_{0,1}$ ) disjoint du bord de $\mathcal{P}_{\infty}$ et recouvert par une partition de type $T$.

Lemme 2.5. - Pour tous $p$ et $q$ (avec $q \geqslant p+1$ ), si un point $\pi(y, s)$ est dans $\mathcal{P}_{p, q}$ et s'il existe un temps $t$ positif tel que $X^{t}(\pi(y, s))$ ne soit plus dans $\mathcal{P}_{p, q}$, alors pour tout $t^{\prime}$ plus grand que $t, X^{t^{\prime}}(\pi(y, s))$ n'est pas dans $\mathcal{P}_{p, q}$.

Autrement dit, toute orbite périodique qui intersecte $\mathcal{P}_{p, q}$ est contenue dans $\mathcal{P}_{p, q}$ et l'intersection de toute orbite avec $\mathcal{P}_{p, q}$ est connexe.

Démonstration. - Rappelons que, par définition de la relation d'équivalence $\sim$, un point $\pi(x, u)$ appartient à $\mathcal{P}_{p, q}$ si et seulement si il existe un entier $k$ tel que $u-k \in[p, q]$ et tel que $\varphi^{k}(x)$ soit défini.

Ceci nous permet tout d'abord pour fixer les idées, de supposer $s \in$ $[p, q]$, cela quitte à échanger $(y, s)$ pour un autre couple dans sa classe d'équivalence.

Ceci fait, il existe, par hypothèse, un temps $t$ positif tel que $X^{t}(\pi(y, s))=$ $\pi(y, s+t)$ n'est pas dans $\mathcal{P}_{p, q}$. Ceci implique que $\varphi^{k}(y)$ n'est pas défini dès que $k$ est assez grand pour que $s+t-k \leqslant q$. Mais, pour tout $t^{\prime}>t$, si $k$ est assez grand pour que $s+t^{\prime}-k \leqslant q$ alors $k$ est a fortiori assez grand 
pour que $s+t-k$ soit plus petit que $q$ et $\varphi^{k}(y)$ n'est donc pas défini. On en déduit que, pour tout $t^{\prime}>t, X^{t^{\prime}}(\pi(y, s))=\pi\left(y, s+t^{\prime}\right)$ n'est pas dans $\mathcal{P}_{p, q}$.

Par un argument usuel, cette propriété pour les orbites positives implique la même propriété pour les orbites négatives.

On notera $K$ le maximal invariant de $\mathcal{P}_{0,1}$ sous l'action du flot de $X$.

LEMME 2.6

$-K$ est égal au maximal invariant de $\mathcal{P}_{p, q}$ pour tous $p \leqslant 0$ et tout $q \geqslant 1$,

- $K$ contient l'ensemble non errant de $\mathcal{P}_{\infty}$ pour le flot de $X$.

$-K$ est contenu dans l'intérieur de $\mathcal{P}_{0,1}$ (on considère l'intérieur de $\mathcal{P}_{0,1}$ comme variété à bords et non pas comme sous-ensemble de $\left.\mathcal{P}_{\infty}\right)$,

- Tout d'abord, soit $\pi(x, t)$ un point du maximal invariant de $\mathcal{P}_{p, q}$. On doit montrer que $\pi(x, t)$ est dans le maximal invariant de $\mathcal{P}_{0,1}$. Pour tout $s, X^{s}(\pi(x, t))=\pi(x, t+s)$ est dans $\mathcal{P}_{p, q}$ par hypothèse. On en déduit que $\varphi^{k}(x)$ est défini pour des $k$ arbitrairement grands et arbitrairement petits et donc pour tout $k$ dans $\mathbb{Z}$. Par conséquent, pour tout $s$, on peut réécrire $X^{s}(\pi(x, t))$ comme $\pi\left(\varphi^{[t+s]}(x), t+s-[t+s]\right)$ (où [.] désigne la partie entière), ce qui signifie que, pour tout $s, X^{s}(\pi(x, t))$ est dans $\mathcal{P}_{0,1}$. Le maximal invariant de $\mathcal{P}_{p, q}$ est donc inclus dans celui de $\mathcal{P}_{0,1}$.

- Soit maintenant $\pi(x, t)$ un point qui n'est pas dans $K$. On veut montrer que $\pi(x, t)$ est errant. Soient $p$ et $q$ tels que $\pi(x, t) \in \mathcal{P}_{p, q}$.

D'après la première affirmation du lemme, l'orbite, disons positive pour fixer les idées, de $\pi(x, t)$ sort de chacun des $\mathcal{P}_{p^{\prime}, q^{\prime}}$, en particulier de $\mathcal{P}_{p-3, q+3}$, disons au bout d'un temps $T$. Par continuité du flot, il en va de même pour un voisinage $U$ de $\pi(x, t)$. D'après la proposition 2.4, on peut choisir $U$ dans $\mathcal{P}_{p-3, q+3}$.

On déduit du lemme 2.5 que l'orbite positive de $X^{T}(U)$ ne revient plus dans $\mathcal{P}_{p-3, q+3}$ et est donc disjointe de $U$. On en déduit que $\pi(x, t)$ est errant.

- Enfin, voyons la dernière affirmation du lemme. Pour tout point $\pi(x, t)$ du maximal invariant de $\mathcal{P}_{0,1}$, on sait que $\varphi(x)$ et $\varphi^{-1}(x)$ sont définis et donc que $x$ est dans $H \cap V$. Mais, $\varphi^{2}(x)$ est également défini, donc $\varphi(x)$ est dans un sous-rectangle horizontal $H_{k}^{l}$ ce qui implique que $x$ n'est pas dans le bord horizontal d'un $H_{i}^{j}$. De même, $x$ n'est pas dans le bord vertical d'un $V_{k}^{l}$. Ceci implique que $x$ est dans l'intérieur de $H \cap V$. 
On en déduit que $\pi(H \times[-\varepsilon, 1+\varepsilon]) \cap \pi(V \times[-\varepsilon, 1+\varepsilon])$ est un voisinage de $K$. Et on vérifie, grâce aux identifications, que cet ensemble est inclus dans $\pi(H \times[0,1]) \cup \pi(V \times[0,1])$ et donc dans l'intérieur de $\mathcal{P}_{0,1}$.

La proposition suivante découle de la construction :

Proposition 2.7. - L'ensemble $K$ est un ensemble selle saturé et $\mathcal{R}=\left\{\pi\left(R_{i} \times\{0\}\right)\right\}_{i=1 \ldots n}$ est une partition de Markov essentielle, de type géométrique $T$, de $K$.

Démonstration. - L'hyperbolicité de $K$ provient des propriétés de dilatation et de contraction de l'application $\varphi$ du patron.

Le choix de rectangles disjoints et les propriétés de contraction et de dilatation des feuilletages assure que le maximal invariant $K$ de $\mathcal{P}_{0,1}$ est transversalement totalement discontinu. C'est donc un ensemble selle.

L'ensemble $K$ est saturé dans $\mathcal{P}_{\infty}$ : en effet, d'après le lemme 2.5 , l'orbite d'un point $x$ de $W^{s}(K) \cap W^{u}(K)$ doit être entièrement contenue dans $\mathcal{P}_{0,1}$. Le point $x$ est alors dans $K$ par définition de $K$.

Par construction et d'après le lemme $2.5, \mathcal{R}=\left\{R_{i}\right\}$ vérifie les propriétés d'une partition de Markov essentielle de type $T$ de $K$.

\section{LEMME 2.8}

- Les variétés stables et instables de $K$ sont disjointes du bord de $\mathcal{P}_{\infty}$.

- Pour tous $p$ et $q$, les intersections $W^{s}(K) \cap \mathcal{P}_{p, q}$ et $W^{u}(K) \cap \mathcal{P}_{p, q}$ sont égales à $\bigcap_{-\infty}^{0} X^{t}\left(\mathcal{P}_{p, q}\right)$ et à $\bigcap_{0}^{+\infty} X^{t}\left(\mathcal{P}_{p, q}\right)$.

Démonstration. - Le premier point provient de l'invariance du bord de $\mathcal{P}_{\infty}$ par le flot et du fait que $K$ est inclus dans l'intérieur de $\mathcal{P}_{0,1}$.

Tout point de $\bigcap_{-\infty}^{0} X^{t}\left(\mathcal{P}_{p, q}\right)$ a son orbite positive incluse dans $\mathcal{P}_{p, q}$. Son $\omega$-limite est donc formée d'orbites du maximal invariant de $\mathcal{P}_{p, q}$, c'est à dire de $K$. Ceci montre l'inclusion $W^{s}(K) \cap \mathcal{P}_{p, q} \supset \bigcap_{-\infty}^{0} X^{t}\left(\mathcal{P}_{p, q}\right)$.

Un point dont l'orbite positive sort de $\mathcal{P}_{p, q}$, n'y revient pas (lemme 2.5) donc n'appartient pas à la variété stable de $K$, ce qui montre l'inclusion inverse.

Convention. - A partir de maintenant, pour simplifier les notations, puisque nous n'aurons plus besoin du patron $P$ proprement dit, nous allons identifier $R, H, V, \ldots$ à leur plongements $\pi(R \times\{0\}), \pi(H \times\{0\}), \pi(V \times\{0\})$ 
dans $\mathcal{P}_{\infty}$. Pour un point $x$ de $R$, on parlera donc de l'image de $x$ par le temps $t$ du flot de $X$.

\section{De l'ouvrage $\mathcal{P}_{\infty}$ au modèle de $T$}

Pour tout type géométrique $T$, nous avons construit une variété (non compacte) à bord $\mathcal{P}_{\infty}$ munie d'un flot possédant un ensemble selle $K$ qui admet une partition essentielle de type $T$. On veut découper dans $\mathcal{P}_{\infty}$ un voisinage de $K$ par une variété compacte à bord qui puisse être complétée simplement ensuite en un modèle de $T$. On doit commencer par trouver dans $\mathcal{P}_{\infty}$ des surfaces d'entrée et de sortie que chaque orbite ne coupe au plus qu'une fois.

\subsection{Construction de surfaces d'entrée et de sortie}

On veut construire une paire de surfaces d'entrée sortie, c'est à dire deux surfaces $E$ et $S$ dans $\mathcal{P}_{\infty}$, compactes à bords, disjointes, telles que :

- toute orbite de $\mathcal{P}_{\infty} \backslash W^{s}(K)$ coupe $S$ en un point et un seul et toute orbite de $\mathcal{P}_{\infty} \backslash W^{u}(K)$ coupe $E$ en un point et un seul,

- la surface $S$ est dans le futur de tout point de $\mathcal{P}_{0,1} \backslash W^{s}(K)$ et la surface $E$ est dans le passé de tout point de $\mathcal{P}_{0,1} \backslash W^{u}(K)$.

Remarque. - Sous ces conditions, $E$ est disjointe de $W^{u}(K)$ et $S$ est disjointe de $W^{s}(K)$. En effet, tout point dont l'orbite positive sort de $\mathcal{P}_{0,1}$ n'est pas dans $W^{s}(K)$ et tout point dont l'orbite négative sort de $\mathcal{P}_{0,1}$ n'est pas dans $W^{u}(K)$ : ceci est une conséquence du lemme 2.5 .

Rappelons que $R$ est l'union des rectangles $R_{i} \times\{0\}$ plongés dans $\mathcal{P}_{\infty}$ selon la convention ci-dessus. L'orbite de tout point de $\mathcal{P}_{\infty}$ coupe $R$. Pour construire une paire de surface d'entrée-sortie, il suffit donc de trouver deux surfaces $E$ et $S$ telles que :

- l'orbite positive de tout point de $R \backslash W^{s}(K)$ coupe $S$ en un point et un seul, cela après un temps supérieur à 1 , un seul.

- l'orbite négative de tout point de $R \backslash W^{u}(K)$ coupe $E$ en un point et

Nous allons à présent construire $S$ :

Rappelons que $H$ est l'union des sous-rectangles horizontaux $H_{i}^{j}$ des $R_{i}$ sur lesquels l'application de premier retour sur $R$ (qui coïncide avec le temps 1 du flot) est définie. Considérons $A=R \backslash H=R \backslash X^{-1}(R)$. C'est une union de sous-rectangles horizontaux semi-ouverts des $R_{i}$ (c'est-à-dire 
des sous-rectangles des $R_{i}$ privés de certains de leurs côtés). Il y en a $h_{i}+1$ dans le rectangle $R_{i}$.

On notera $\bar{A}$ l'adhérence de $A$. La différence $\bar{A} \backslash A$ est formée des côtés horizontaux de $\bar{A}$ qui sont aussi dans $H$ (c'est-à-dire les côtés horizontaux de $\bar{A}$ qui ne sont pas dans le bord de $R$ ).

Il sera parfois commode de numéroter les sous-rectangles constituants $A: A_{i}^{0}, \cdots, A_{i}^{h_{i}}$ dans l'ordre de l'orientation des verticales, de $A_{i}^{0}$ en dessous de $H_{i}^{1} \grave{\text { à }} A_{i}^{h_{i}}$ au dessus de $H_{i}^{h_{i}}$.

LEMME 3.1

- L'orbite positive (ou nulle) de tout point de $R \backslash W^{s}(K)$ coupe A.

- L'orbite strictement positive de tout point de $\bar{A} \backslash A$ coupe $A$.

- L'orbite strictement positive de tout point de $A$ ne recoupe pas $A$.

\section{Démonstration}

- On a $W^{s}(K) \cap R=\bigcap_{\mathbb{N}} X^{-n}(R)$ : la démonstration est analogue à celle du lemme 2.8. Par conséquent, pour tout point de $R \backslash W^{s}(K)$, il existe un dernier $n$ positif tel que $X^{n}(x)$ soit dans $R$ et donc soit dans $R \backslash X^{-1}(R)=A$.

- La formule $W^{s}(K) \cap R=\bigcap_{\mathbb{N}} X^{-n}(R)$ indique que $W^{s}(K)$ est disjoint de $A$ donc de $\partial^{s} R$. Par invariance, on en déduit que $W^{s}(K)$ est également disjoint de $\partial^{s} X^{-1}(R) \cap R=\partial^{s} H=\bar{A} \backslash A$. On conclut grâce au premier point du lemme.

- Le troisième découle du fait que la partition est essentielle : $H$ est le domaine de définition de l'application de premier retour sur $R$.

L'orbite positive de tout point de $R \backslash W^{s}(K)$ coupe $\bar{A}$. Cependant, $\bar{A}$ n'est pas encore la surface de sortie recherchée : le problème est que certaines orbites coupent deux fois $\bar{A}$ (les orbites ne coupent qu'une seule fois $A$, mais on veut une surface compacte). Les points de $\bar{A}$ dont l'orbite positive recoupe $\bar{A}$ sont situés sur $\bar{A} \backslash A$ et leur retour sur $\bar{A}$ est situé sur $\bar{A} \cap \partial^{s} R$. Pour obtenir une surface de sortie, nous allons pousser $\bar{A} \backslash A$ le long du flot de façon à le recoller à son image par l'application de premier retour. Précisément :

Considérons $\psi$ une fonction $C^{\infty}$ définie sur $\bar{A}$ à valeur réelles, valant 1 sur un voisinage de $A \cap \partial^{s} R$, valant 2 sur un voisinage $\bar{A} \backslash A$ et telle que $1 \leqslant \psi(x) \leqslant 2$. 
Considérons maintenant :

$$
S=\bigcup_{x \in \bar{A}} X^{\psi(x)}(x)
$$

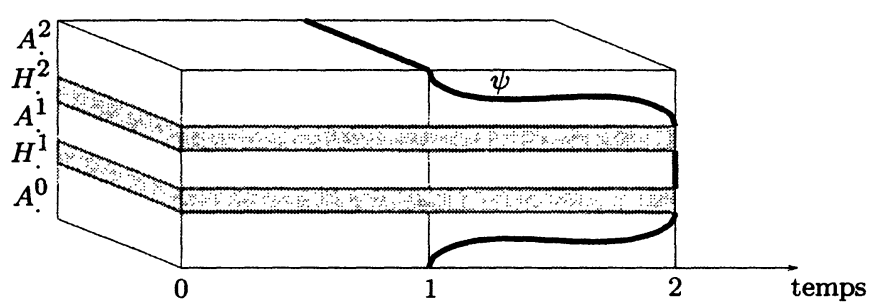

Fig. 5 La fonction $\psi$

Proposition 3.2. - L'orbite positive de tout point $x$ de $R \backslash W^{s}(K)$ coupe $S$ en un point $X^{t(x)}(x)$ et un seul. De plus, le temps $t(x)$ est supérieur ou égal à 1 .

Démonstration. - D'après ce qui précède, il suffit de montrer que l'orbite de tout point de $\bar{A}$ coupe $S$ en un point et un seul, ce que nous allons faire.

D'abord, par construction, l'orbite de tout point $x$ de $\bar{A}$ coupe $S$ en $X^{\psi(x)}(x)$. D'autre part, on a une projection via le flot de $X$, de $\bar{A}$ sur $S$ donc toute orbite qui coupe $\bar{A}$ en au plus un point coupe $S$ en au plus un point. Il suffit donc de voir que les orbites qui coupent deux fois $\bar{A}$ ne coupe $S$ qu'une seule fois.

Mais une orbite qui coupe $\bar{A}$ deux fois le fait en $x \in \bar{A} \backslash A$ et en $y=$ $X^{1}(x) \in A \cap \partial^{s} R$. Cette orbite coupe donc $S$ en $X^{\psi(x)}(x)=X^{2}(x)$ et en $X^{\psi(y)}(y)=X^{1}(y)=X^{2}(x)$, donc en un seul point. De plus, $\psi(x) \geqslant 1$, ce qui achève la démonstration.

Proposition 3.3. - L'ensemble $S$ est une surface à bord, arêtes et coins, transverse $\grave{a} X$.

Démonstration. - Par construction de $\mathcal{P}_{\infty}$, deux points $X^{t_{1}}\left(x_{1}\right)$ et $X^{t_{2}}\left(x_{2}\right)$ où $x_{1}$ et $x_{2}$ sont dans $R$ ne peuvent être égaux que si $t_{1}-t_{2} \in \mathbb{Z}$.

On en déduit que $S$ est homéomorphe à $\bar{A}$ quotienté par l'application de premier retour de $X$ sur $\bar{A}$ (cette application coïncide avec le temps $1 \mathrm{du}$ flot). Cette application est définie sur les côtés horizontaux formant $\bar{A} \backslash A$ et à valeur dans les côtés horizontaux formant $\bar{A} \cap \partial^{s} R$.

L'ensemble $S$ est donc obtenu à partir d'une union finie de rectangles compacts disjoints en recollant une famille finie de segments disjoints, inclus 
dans le bord de ces rectangles, par un difféomorphisme, sur une famille finie de segments disjoints, inclus dans le bord de ces rectangles, cette dernière famille étant disjointe de la précédente. C'est donc une surface compacte à bord et coins.

La transversalité de $S$ à $X$ découle de la transversalité de $\bar{A}$ à $X$ et de ce qu'on a obtenu $S$ en poussant $\bar{A}$ le long du flot pendant un temps différentiable $\psi$.

La structure différentiable de $S$ le long des recollements provient du choix $\psi=1$ (et $\psi=2$ ) sur des voisinages des côtés horizontaux de $\bar{A}$.
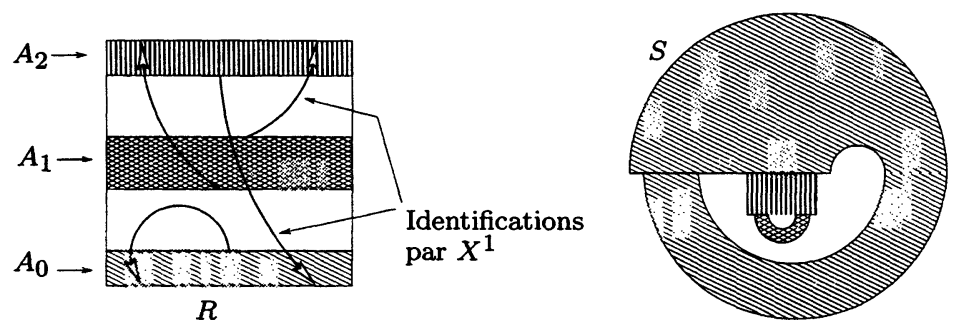

Fig. 6 Construction de la surface $S$ pour le type géométrique à un rectangle du fer à cheval

Remarque. - La preuve de 3.3 donne un procédé de construction de la surface $S$ par recollement de rectangles le long de segments de leurs bords, l'ordre de ces segments étant donné par le type géométrique $T$. Il est d'abord clair que $S$ ne dépend pas (à difféomorphisme près) de la fonction $\psi$ choisie. Ensuite, ceci donne un procédé de calcul algorithmique du genre de $S:$ Il suffit alors de se rappeller que le genre d'un disque sur lequel ont a recollé des anses sur des segments en ordre prescrit est algorithmiquement calculable et de se ramener à ce cas.

DÉFINITION. - On dira que $S$ est la surface de sortie associée au type géométrique $T$.

Une construction analogue permet d'obtenir une surface d'entrée $E$ telle que l'orbite négative de toute point de $R \backslash W^{u}(K)$ coupe $E$ en un point et un seul ; cela en remplaçant les sous-rectangles horizontaux par les sousrectangles verticaux et les temps positifs du flot de $X$ par les mêmes temps négatifs. 


\subsection{Découpage de $\mathcal{P}_{\infty}$ et colmatage des trous}

Nous venons de construire une paire de surface d'entrée-sortie $E$ et $S$. Ceci nous permet de définir un temps de sortie $T^{S}$ : pour tout point $x$ de $\mathcal{P}_{\infty} \backslash W^{s}(K), T_{x}^{S}$ est l'unique temps tel que $X^{T_{x}^{S}}(x)$ appartient à $S$ et on étend $T_{x}^{S}$ sur $W^{s}(K)$ par $+\infty$. De même, on définit le temps d'entrée $T^{E}$ sur $\mathcal{P}_{\infty} \backslash W^{u}(K)$ (en remplaçant $S$ par $E$ ) et on l'étend par $-\infty$ sur $W^{u}(K)$. Rappelons que les temps $T_{x}^{S}$ et $T_{x}^{E}$ sont respectivement positifs et négatifs $\operatorname{sur} \mathcal{P}_{0,1}$.

LEMME 3.4. - Les temps $T_{x}^{S}$ et $T_{x}^{E}$ sont des fonctions continues de $x$ à valeurs dans $\mathbb{R} \cup\{ \pm \infty\}$. Ce sont même des fonctions de classe $C^{1}$ respectivement sur $\mathcal{P}_{\infty} \backslash W^{s}(K)$ et $\mathcal{P}_{\infty} \backslash W^{u}(K)$.

Démonstration. - On fait la démonstration pour le temps de sortie $T_{x}^{S}$. Au voisinage d'un point $x$ dont le temps de sortie $T_{x}^{S}$ est fini et dont le point de sortie $X^{T_{x}^{S}}(x)$ est dans l'intérieur de $S$, la continuité du temps de sortie est simplement dûe à la transversalité de $S$ à $X$. Cependant il pourrait exister des difficultés au voisinage des points qui ne sortent pas (c'est à dire au voisinage de $W^{s}(K)$ ) et au voisinage des points qui sortent dans le bord de $S$. C'est pourquoi nous devons distinguer plusieurs cas.

Soit $x_{n}$ une suite de points convergeant vers $x$. On notera $T_{n}=T_{x_{n}}^{S}$.

Remarquons tout d'abords que les temps $T_{n}$ restent minorés : d'après le lemme 2.4, il existe $p \leqslant 0$ et $q \geqslant 2$ finis tels que, pour $n$ assez grand, $x_{n}$ est dans l'ouvrage $\mathcal{P}_{p, q}$. Par construction, $S$ est incluse dans $\mathcal{P}_{1,2}$ et donc dans $\mathcal{P}_{p, q}$. Les $T_{n}$ sont alors supérieurs à $-q$. En effet, pour tout point de $\mathcal{P}_{p, q}$ il existe $t \geqslant-q$ tel que $y=X^{t}(x) \in R$; le temps $T_{x}^{S}$ est alors égal à $t+T_{y}^{S}$ avec $T_{y}^{S}>0$ par construction de $S$.

Maintenant, quitte à extraire une sous-suite, on peut supposer que les $T_{n}$ convergent vers $T$ appartenant à $\mathbb{R} \cup\{+\infty\}$. Nous allons montrer que dans ce cas, $T$ est égal à $T_{x}^{S}$.

- Supposons d'abord $T$ fini. Alors, par continuité du flot et compacité de $S$, on a $X^{T}(x) \in S$. L'unicité du temps de sortie de $x$ implique que $T_{x}^{S}=T$.

- Supposons alors $T=+\infty$ et supposons d'abord que les $T_{n}$ sont finis. Puisque $x_{n}$ et $X^{T_{n}}\left(x_{n}\right)$ appartiennent à $\mathcal{P}_{p, q}$, le lemme 2.5 implique que le segment d'orbite joignant ces deux points est inclus dans $\mathcal{P}_{p, q}$. En d'autres termes, $x_{n} \in \bigcap_{-T_{n}}^{0} X^{t}\left(\mathcal{P}_{p, q}\right)$. Comme $T_{n} \rightarrow+\infty$, on a que $x \in$ $\bigcap_{-\infty}^{0} X^{t}\left(\mathcal{P}_{p, q}\right)$, c'est à dire que $x \in W^{s}(K)$. Par conséquent, $T_{x}^{S}=+\infty=T$. 
- Quitte à prendre une sous-suite, il reste le cas où les $T_{n}$ valent uniformément $+\infty$, c'est à dire que $x_{n} \in W^{s}(K) \cap \mathcal{P}_{p, q}$. On a vu que la variété stable $W^{s}(K) \cap \mathcal{P}_{p, q}$ est égale à $\bigcap_{-\infty}^{0} X^{t}\left(\mathcal{P}_{p, q}\right)$ donc est compacte. Donc $x \in W^{s}(K)$ et $T_{x}^{S}=+\infty=T$.

Dans tous les cas, on a montré que $T_{x}^{S}=\lim T_{x_{n}}^{S}$. La différentiabilité de $T_{x}^{S}$ sur $\mathcal{P}_{\infty} \backslash W^{s}(K)$ provient de la transversalité de $X$ à $S$.

Corollaire 3.5. - Les bords $\partial E$ et $\partial S$ de $E$ et de $S$ sont inclus dans le bord de $\mathcal{P}_{\infty}$.

Démonstration. - Il suffit de voir qu'un point du bord de $S$ (respectivement de $E$ ) dans l'intérieur de $\mathcal{P}_{\infty}$ serait un point de discontinuité de $T^{S}$ (respectivement de $T^{E}$ ).

Considérons $M^{0}$ la variété obtenue en découpant $\mathcal{P}_{\infty}$ le long de $E$ et $S$; formellement :

$$
M^{0}=\left\{x \in \mathcal{P}_{\infty} \text { tels que } T_{x}^{S} \in \mathbb{R}^{+} \cup\{+\infty\} \text { et } T_{x}^{E} \in \mathbb{R}^{-} \cup\{-\infty\}\right\}
$$

PROPOSITION 3.6. - L'ensemble $M^{0}$ est une variété compacte à bords et arêtes et coins de dimension 3 contenant $K$ dans son intérieur.

L'intersection de $M^{0}$ avec toute orbite de $X$ est connexe.

Le bord de $M^{0}$ est formé de :

- une surface d'entrée $E$ et une surface de sortie $S$, qui sont transverses au flot et qui intersectent chacune toute orbite en au plus un point,

- un nombre fini de composantes tangentes au champ, dont l'union, appelée bord tangent, est égale à l'intersection de $M^{0}$ avec le bord de $\mathcal{P}_{\infty}$. Ce bord tangent est aussi l'orbite dans $M^{0} d u$ bord de $E$ (ou de $S$ ). De plus, il existe un difféomorphisme du bord tangent de $M^{0}$ sur $\partial E \times[0,1]$ réalisant une équivalence entre le champ $X$ et le champ $\frac{\partial}{\partial t}$ de $\partial E \times[0,1]$ (on place des coordonnées $(x, t)$ sur le produit cartésien $\partial E \times[0,1])$.

On a représenté aux figures 7 et 8 les variétés à bords $\mathcal{P}_{1,2}$ puis $M^{0}$ obtenues dans le cas le plus simple possible : $K$ est réduit à une orbite périodique selle sans réflexion.

La figure 9 donne une représentation de la variété $M^{0}$ obtenue dans un cas plus compliqué : le type géométrique utilisé est celui de la partition à un rectangle du fer à cheval de Smale usuel. Cependant de nombreuses arêtes et coins de $M^{0}$ ont été supprimés pour rendre le schéma possible. 


\section{Construction de flots de Smale en dimension 3}
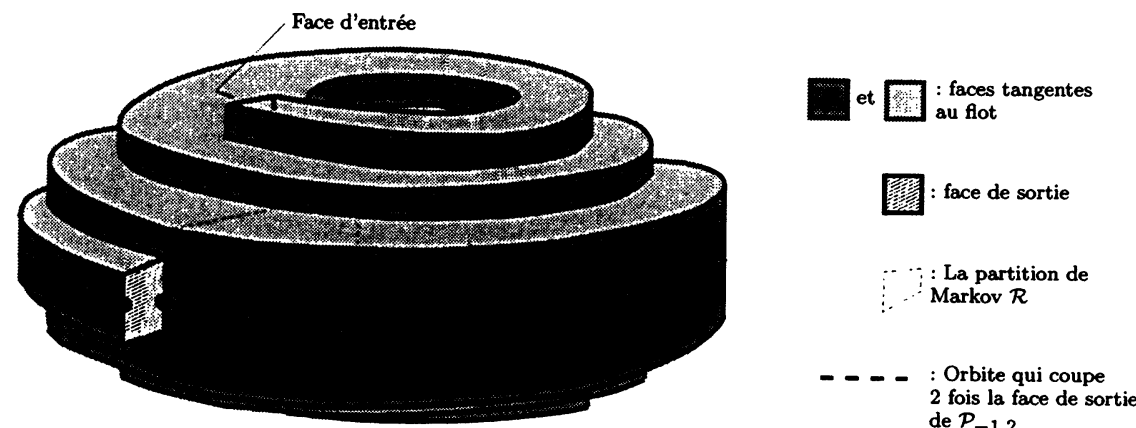

Fig. 7 La variété à bord $\mathcal{P}_{1,2}$ dans le cas où $K$ est réduit à une seule orbite périodique selle
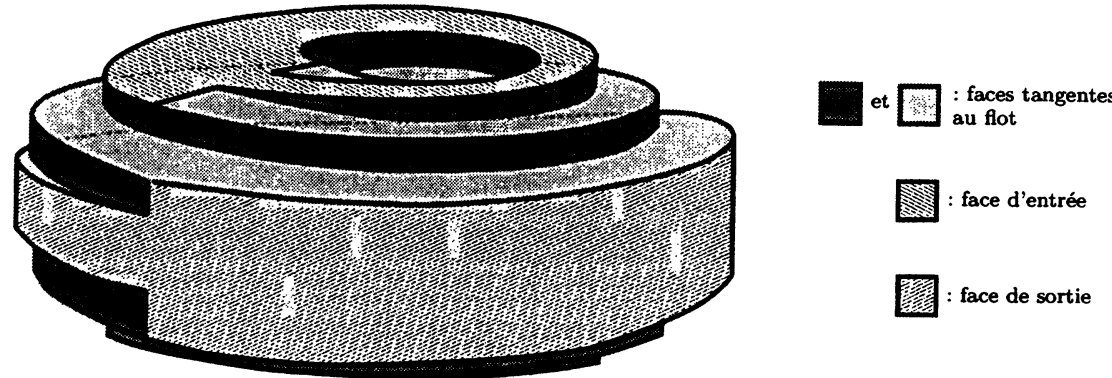

Fig. 8 La variété à bord $M^{0}$ obtenue par découpage de $\mathcal{P}_{1,2}$ dans le cas où $K$ est réduit à une seule orbite périodique selle

Démonstration de la proposition 3.6 - Par construction de $E$ et $S$, $M^{0}$ est incluse dans la variété compacte $\mathcal{P}_{-2,2}$. La continuité de $T^{E}$ et $T^{S}$ montre que l'extraction de $M^{0}$ consiste effectivement à découper $\mathcal{P}_{-2,2}$ le long de $E$ et $S$, et à ne conserver que les composantes connexes rencontrant $K$. On en déduit, grâce à 3.5 , que $M^{0}$ est une variété compacte à bord, arêtes et coins. La structure différentielle provient de ce que $E$ et $S$ sont transverses au flot.

L'ensemble $K$ correspond au lieu où $T^{E}=-\infty$ et $T^{S}=+\infty$ donc est inclus dans $M^{0}$. L'analyse suivante montre que $K$ est disjoint des différents bords de $M^{0}$ et est donc inclus dans son intérieur.

On voit de même que $E$ et $S$ sont incluses dans le bord de $M^{0}$. L'intersection de l'orbite de tout point $x$ avec $M^{0}$ correspond à l'intervalle de temps $\left[T_{x}^{E}, T_{x}^{S}\right]$, donc est connexe. 


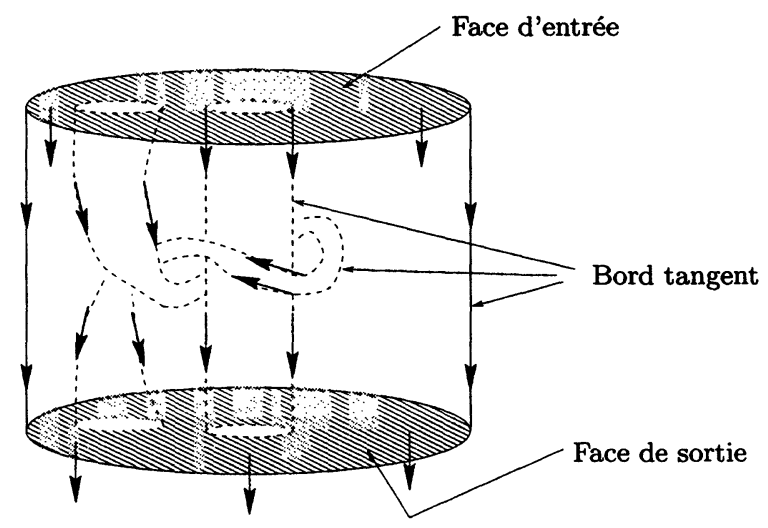

Fig. 9 La variété $M^{0}$ obtenue pour la partition à un rectangle du fer à cheval usuel (avec certaines arêtes et certains coins supprimés)

Le bord de $M^{0}$ dans $\mathcal{P}_{\infty}$ est égal au lieu où $T^{E}=0$ ou $T^{S}=0$, c'està-dire à $E \cup S$. On en déduit que le bord de $M^{0}$ privé de $E$ et $S$ est inclus dans le bord de $\mathcal{P}_{\infty}$ donc est tangent au champ $X$. De plus, le bord de $\mathcal{P}_{\infty}$ est disjoint des variétés invariantes de $K$, toute orbite du bord de $\mathcal{P}_{\infty}$ coupe donc les bords $\partial E$ et $\partial S$ en un et un seul point. L'intersection d'une orbite avec $M^{0}$ étant connexe, toute orbite de $\partial \mathcal{P}_{\infty}$ coupe $M^{0}$ en un segment joignant $\partial E$ à $\partial S$. Le difféomorphisme annoncé consiste simplement à prendre le flot du champ $X$ renormalisé de façon à ce que le temps de sortie d'un point de $\partial E$ soit égal à 1.

Nous allons maintenant tuer les bords tangents de $M^{0}$ et terminer ainsi la construction d'un voisinage de $K$ par une variété à bord transverse à $X$ :

Chaque composante $C$ du bord de $E$ est un cercle ; son orbite est une composante du bord tangent de $M^{0}$ difféomorphe à un cylindre $C \times[0,1]$ joignant $C$ à une composante du bord de $S$. Le champ $X$ le long de cette composante de bord est équivalent, via ce difféomorphisme, au champ trivial $\frac{\partial}{\partial t}$ de $C \times[0,1]$.

Pour chaque telle composante $C$, on choisit alors un disque $D$ dont le bord est difféomorphe à $C$. Puis, on colle sur l'orbite de $C$ le cylindre plein $D \times[0,1]$ muni du champ $\frac{\partial}{\partial t}$ via un difféomorphisme qui recolle les champs.

D'après la proposition 3.6, on supprime par ce procédé toute les composantes du bord de $M^{0}$ tangentes au champ $X$. On obtient donc une variété compacte à bord $M$, munie d'une extension de $X$ (que nous appellerons toujours $X$ ) transverse au bord. Le bord de $M$ est constitué d'un bord d'entrée 
$E^{\prime}$, difféomorphe à la surface $E$ sur chaque composante de bord de laquelle on a recollé un disque et d'un bord de sortie $S^{\prime}$, obtenu lui-aussi en recollant des disques sur le bord de $S$.

Les cylindres pleins recollés étant munis du champ $\frac{\partial}{\partial t}$, il est clair que $K$ est le maximal invariant de $M$. Les propriétés de $K$ (hyperbolicité, saturation,..) sont évidemment toujours vérifiées dans $M$. Comme cela était déjà vrai dans $M^{0}$, toute composante de $M$ rencontre $K$.

En résumé, on a donc construit $M$ tel que :

Proposition 3.7. - $M$ est une variété compacte, à bord, de dimension 3, munie d'un champ $X$ transverse au bord. Le maximal invariant de $M$ est un ensemble selle saturé $K$ qui admet la partition de Markov essentielle $\mathcal{R}$ de type géométrique $T$. Enfin, toute composante connexe de $M$ rencontre $K$.

\subsection{Le couple $(M, X)$ construit est un modèle de $T$}

Pour montrer que $M$ est un modèle de $T$ et donc montrer le théorème 0.1 et le corollaire 0.2 , il suffit, d'après la proposition 3.7 , de prouver que, pour tout cercle $C$ plongé dans le bord d'entrée $\partial_{1} M$ et disjoint de $W^{s}(K), C$ borde dans $\partial_{1} M$ un cercle disjoint de $W^{s}(K)$.

Proposition 3.8. - Notons $\partial_{1} M$ le bord d'entrée de $X$ dans $M$. Tout cercle $C$ plongé dans $\partial_{1} M$ et disjoint de $W^{s}(K)$ borde, dans $\partial_{1} M$, un disque disjoint de $W^{s}(K)$. En d'autres termes, $(M, X)$ est un modèle de $T$.

Remarque. - Rappelons que la variété $M$ a été construite en recollant des cylindres pleins sur le bord tangent de $M^{0}$. La variété à bord $M^{0}$ peut alors être vue comme le saturé dans $M$ par le flot de $X$ de l'union des rectangles de la partition $\mathcal{R}$.

Si $C$ est un cercle plongé dans $\partial_{1} M$ et disjoint du saturé des rectangles de $\mathcal{R}$, c'est à dire de $M^{0}$, alors $C$ borde un disque sur $\partial_{1} M$, ce disque étant disjoint de $M^{0}$ et donc de $W^{s}(K)$.

La démonstration de la proposition 3.8 va consister a approcher $W^{s}(K) \cap$ $\partial_{1} M$ par l'intersection avec $\partial_{1} M$ du saturé des rectangles d'une petite partition de Markov obtenue en subdivisant la partition $\mathcal{R}$. Nous allons montrer que la propriété de la remarque ci-dessus se propage à des subdivisions successives de $\mathcal{R}$, pourvu que les partitions considérées soient essentielles. L'étape fondamentale de la démonstration est constituée des lemmes 3.9 et 3.10 . 
LEMME 3.9. - Soit $\tilde{\mathcal{R}}$ une partition essentielle de $K$ dans $M$ et soit $\tilde{\mathcal{R}}^{\prime}$ la partition dont les rectangles sont les sous-rectangles horizontaux de $\tilde{\mathcal{R}}, c^{\prime}$ est-à-dire sont les composantes connexes du domaine de définition de l'application de premier retour.

Alors $\tilde{\mathcal{R}}^{\prime}$ est également essentielle.

Démonstration. - On remarque que le premier retour des rectangles de $\tilde{\mathcal{R}}^{\prime}$ sur la transversale $\tilde{R}$ (l'union des rectangles de $\tilde{\mathcal{R}}$ ) est bien définie. Il suffit alors de noter que les points de retour sur $\tilde{R}$ qui ne sont pas dans $\tilde{R}^{\prime}$ n'ont aucun retour dans $\tilde{R}^{\prime}$. En effet, ils n'ont aucun retour dans $\tilde{R}$ car $\tilde{\mathcal{R}}$ est essentielle.

Pour toute partition $\mathcal{S}$ de $K$ dans $M$, on notera $\operatorname{Sat}(\mathcal{S})$ le saturé par le flot de $X$ de l'union des rectangles de $\mathcal{S}$ dans $M$. De plus, on dira que la partition $\mathcal{S}$ vérifie la propriété $(*)$, si :

$\left.{ }^{*}\right)$ pour tout cercle $C$ sur $\partial_{1} M$ disjoint de $\operatorname{Sat}(\mathcal{S})$, le cercle $C$ borde un disque sur $\partial_{1} M$ disjoint $\operatorname{de} \operatorname{Sat}(\mathcal{S})$

LEMME 3.10. - Soit $\tilde{\mathcal{R}}$ une partition essentielle de $K$ dans $M$ possédant la propriété $\left(^{*}\right)$ ci-dessus. Soit $\tilde{\mathcal{R}}^{\prime}$ la partition dont les rectangles sont les sous-rectangles horizontaux de $\tilde{\mathcal{R}}$.

Alors $\tilde{\mathcal{R}}^{\prime}$ possède aussi la propriété (*).

Démonstration. - Notons $\tilde{R}$ et $\tilde{R}^{\prime}$ les unions des rectangles des partitions de Markov $\tilde{\mathcal{R}}$ et $\tilde{\mathcal{R}}^{\prime}$. On notera également $\tilde{H}$ et $\tilde{V}$ les unions des sous-rectangles horizontaux et verticaux de $\tilde{\mathcal{R}}$. Par définition, on a $\tilde{R}^{\prime}=\tilde{H}$.

Dans cette démonstration, nous utiliserons les remarques suivantes :

Remarques.

i) Pour tout compact $D$ de $M$, disjoint des variétés invariantes de $K$ et tel que pour tous temps $t_{1}$ et $t_{2}$ distincts, $X^{t_{1}}(D) \cap X^{t_{2}}(D)$ soit vide, l'orbite de $D$ est topologiquement équivalente à $\left(D \times[0,1], \frac{\partial}{\partial t}\right)$. En effet, la projection le long des orbites de $D$ sur le bord d'entrée $\partial_{1} M$ est continue et injective donc est un homéomorphisme. Il suffit alors de renormaliser le temps de sortie.

ii) Pour tous temps $t_{1}$ et $t_{2}$ distincts, l'intersection $X^{t_{1}}(\tilde{R} \backslash \tilde{H}) \cap X^{t_{2}}(\tilde{R} \backslash$ $\tilde{H})$ est vide. En effet, $\tilde{\mathcal{R}}$ étant essentielle, pour tout $x$ dans $\tilde{R} \backslash \tilde{H}$ et tout $t$ strictement positif, $X^{t}(x)$ n'est pas dans $\tilde{R}$.

iii) La variété stable locale $W^{s}(K) \cap \tilde{R}$ est incluse dans $\tilde{H}$. De même, $W^{u}(K) \cap \tilde{R}$ est inclus dans l'union $\tilde{V}$ des sous-rectangles verticaux de $\tilde{\mathcal{R}}$. 
Soit donc $C$ un cercle sur $\partial_{1} M$ disjoint $\operatorname{de} \operatorname{Sat}\left(\tilde{\mathcal{R}}^{\prime}\right)$. Puisque $\tilde{\mathcal{R}}$ vérifie la propriété $\left(^{*}\right)$, si $C$ est disjoint $\operatorname{de} \operatorname{int}(\operatorname{Sat}(\tilde{\mathcal{R}}))$, alors, $C$ borde un disque $\operatorname{disjoint} \operatorname{de} \operatorname{Sat}(\tilde{\mathcal{R}})$ et, a fortiori, $\operatorname{disjoint} \operatorname{de} \operatorname{Sat}\left(\tilde{\mathcal{R}}^{\prime}\right)$.

Sinon, remarquons tout d'abord que l'intersection $\operatorname{Sat}(\tilde{\mathcal{R}}) \cap \partial_{1} M$ est une sous-surface à bords de $\partial_{1} M$ et que l'on peut supposer $C$ en position générique par rapport au bord de cette sous-surface. En effet, le résultat du lemme est indépendant d'une isotopie de $C$ à support disjoint de $\operatorname{Sat}\left(\tilde{\mathcal{R}}^{\prime}\right)$ (qui est compact et disjoint de $C$ ).

On note alors $C^{0}=C \cap \operatorname{Sat}(\tilde{\mathcal{R}})$. D'après la remarque ci-dessus, $C^{0}$ est soit une courbe fermée si $C \subset \operatorname{Sat}(\tilde{\mathcal{R}})$, soit une union finie de segments inclus dans $\operatorname{Sat}(\tilde{\mathcal{R}}) \cap \partial_{1} M$, à extrémités sur le bord de $\operatorname{Sat}(\tilde{\mathcal{R}})$. On note alors $C^{\prime}=a d h\left(C \backslash C^{0}\right)$; c'est cette fois une union finie de segments d'intérieurs disjoints de $\operatorname{Sat}(\tilde{\mathcal{R}})$, et à extrémités sur le bord de $\operatorname{Sat}(\tilde{\mathcal{R}})$.

L'orbite de $C^{0}$ est, d'après le $i$ ) de la remarque précédent la démonstration, un cylindre difféomorphe à $C^{0} \times[0,1]$ le long duquel le champ $X$ est topologiquement équivalent à $\frac{\partial}{\partial t}$. Or, les rectangles $\tilde{R}_{i}$ sont d'une part transverses au champ $X$ et donc au cylindre, et, d'autre part, disjoints du bord de $M$ donc du bord de ce cylindre. On en déduit que l'intersection de l'orbite de $C^{0}$ et des $\tilde{R}_{i}$ est une union de courbes simples, disjointes, compactes et dont les extrémités éventuelles sont situées sur le bord des $\tilde{R}_{i}$. Par un argument de position générale, on peut encore une fois supposer cette union finie. Notons $\gamma_{1}, \ldots, \gamma_{n}$ ces courbes et $\Gamma$ leur réunion.

On sait que $\Gamma$ est disjointe de $\tilde{H}\left(\operatorname{car} C^{0}\right.$ est disjointe $\left.\operatorname{de} \operatorname{Sat}\left(\tilde{\mathcal{R}}^{\prime}\right)\right)$ mais aussi de l'union des sous-rectangles verticaux $\tilde{V}=X^{1}(\tilde{H})$ (voir figure 10).

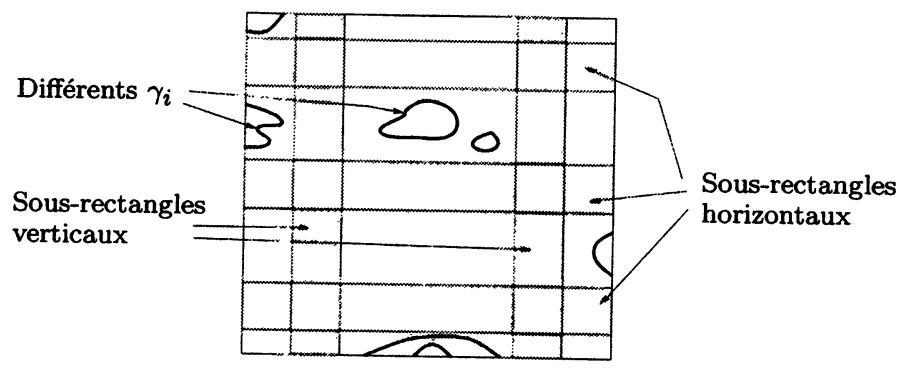

Fig. 10 Position, a priori, des $\gamma_{i}$ 
- Si une des courbes de $\Gamma$ (disons $\gamma_{j}$ incluse dans $\tilde{R}_{i}$ ) est fermée, alors $C$ borde un disque disjoint de $W^{s}(K)$. En effet, $\gamma_{j}$ borde alors un disque $D$ sur un des $\tilde{R}_{i}$. La courbe $\gamma_{j}$ est disjointe des sous-rectangles horizontaux et verticaux de $\tilde{R}_{i}$. Or, ces sous-rectangles rencontrent tous le bord des $\tilde{R}_{i}$, ce qui implique que le disque $D$ est nécessairement disjoint de $\tilde{H}$ et $\tilde{V}$.

Ceci implique, d'une part, que $D$ est disjoint de $W^{s}(K)$ et de $W^{u}(K)$ et, d'autre part, que pour tous $t_{1}$ et $t_{2}$ distincts, $X^{t_{1}}(D) \cap X^{t_{2}}(D)$ est vide (voir les $i i$ ) et $i i i$ ) de la remarque précédent la preuve). Par conséquent, d'après le $i$ ) de la même remarque, l'orbite de $D$ dans $M$ est difféomorphe à $D \times[0,1]$. Vu au travers de ce difféomorphisme, $C$ est inclus dans le bord du disque $D \times\{0\}$ de $\partial_{1} M$, ce disque étant disjoint de $\operatorname{Sat}\left(\tilde{\mathcal{R}}^{\prime}\right)$, ce qui conclut.

- Sinon, $\Gamma$ est une union de segments dans l'union des rectangles $\tilde{R}$, les extrémités de ces segments étant sur le bord des rectangles $\tilde{R}_{i}$ et $\Gamma$ étant disjointe des unions de sous-rectangles horizontaux et verticaux $\tilde{H}$ et $\tilde{V}$. La remarque précédent la preuve implique que les saturés des différents $\gamma_{i}$ sont disjoints.

SOUS-LEMME 3.11. - Il existe une homotopie dans $\tilde{R} \backslash(\tilde{H} \cup \tilde{V})$ entre l'union de segments $\Gamma=\Gamma_{0}$ et une union de segments $\Gamma_{1}$ incluse dans le bord de $\tilde{R}$, l'homotopie $\left(\Gamma_{t}\right)$ se passant à extrémités des segments fixées.

Démonstration. - Pour qu'une telle homotopie $\left(\Gamma_{t}\right)$ existe, il suffit que chaque composante connexe de $\tilde{R} \backslash(\tilde{H} \cup \tilde{V})$ soit simplement connexe et que son intersection avec le bord de $\tilde{R}$ soit connexe et non vide.

Les $\tilde{H}_{i}^{j}$ sont des sous-rectangles horizontaux qui traversent donc les $\tilde{R}_{i}$ de part en part (de gauche à droite) et les $\tilde{V}_{k}^{l}$ sont des sous-rectangles verticaux qui traversent donc les $\tilde{R}_{k}$ de part en part (de bas à haut) ; on en déduit que $\tilde{R} \backslash(\tilde{H} \cup \tilde{V})$ est une union finie de sous-rectangles (semiouverts) d'adhérences disjointes (chaque composante de $\tilde{R} \backslash(\tilde{H} \cup \tilde{V})$ est donc simplement connexe). Dans chaque rectangle $\tilde{R}_{i}$, il y a au moins un $\tilde{H}_{i}^{j}$ et au moins un $\tilde{V}_{i}^{l} ;$ on en déduit que l'intersection d'une composante de $\tilde{R} \backslash(\tilde{H} \cup \tilde{V})$ avec le bord de $\tilde{R}$ est toujours connexe.

Sous-LemMe 3.12. - Pour chaque $s$, l'orbite de $\Gamma_{s}$ est difféomorphe à un cylindre $\Gamma_{s} \times[0,1]$ qui intersecte $\partial_{1} M$ en $\Gamma_{s} \times\{0\}$. De plus, l'intersection du saturé de $\Gamma_{s}$ et de $\tilde{R}$ est égal à $\Gamma_{s}$.

Démonstration. - Ceci provient encore une fois des remarques précédant la preuve.

Fin de la démonstration du lemme 3.10. - L'intersection de l'orbite de $\Gamma^{s}$ et de $\partial_{1} M$ réalise donc une homotopie (disjointe $\operatorname{de} \operatorname{Sat}\left(\tilde{\mathcal{R}}^{\prime}\right)$ et à 
extrémités fixées) entre $C^{0}$ et une union de courbes à mêmes extrémités que $C^{0}$ et incluses dans le bord de $\operatorname{Sat}(\tilde{\mathcal{R}})$. En recollant cette dernière union de courbes à $C^{\prime}$, on obtient un lacet sur $\partial_{1} M$ disjoint de $\operatorname{int}(\operatorname{Sat}(\tilde{\mathcal{R}}))$ et qui est donc homotope à 0 dans $\partial_{1} M \backslash \operatorname{Sat}(\tilde{\mathcal{R}})$. L'homotopie entre $C$ et ce lacet se passant hors de $\operatorname{Sat}\left(\tilde{\mathcal{R}}^{\prime}\right), C$ est donc homotope à 0 dans $\partial_{1} M \backslash \operatorname{Sat}\left(\tilde{\mathcal{R}}^{\prime}\right)$, ce qui conclut.

En appliquant le lemme 3.10 à des raffinements successifs de la partition $R$, on a montré :

Corollaire 3.13. - Soit maintenant $\mathcal{R}^{\prime}$ la partition de $K$ dont les rectangles sont les composantes connexes de $\bigcap_{i=-N}^{P} X^{i}(R)$ (pour un certain $N$ et un certain $P$ fixés).

Alors $\mathcal{R}^{\prime}$ est essentielle et vérifie la propriété $\left({ }^{*}\right)$.

On remarque d'abord que le saturé de $\bigcap_{i=-N}^{P} X^{i}(R)$ est le même que celui de $\bigcap_{i=0}^{P+N} X^{i}(R)$. La démonstration se fait alors par récurrence : passer de $N+P$ à $N+P+1$ revient à appliquer le lemme 3.10.

La démonstration de la proposition 3.8 découle du corollaire :

Démonstration de la proposition 3.8 - Soit $C$ un cercle sur $\partial_{1} M$ disjoint de $W^{s}(K)$. L'orbite positive de tout point de $C$ sort de $M$. Le temps de sortie étant continu et $C$ étant compact, ce temps de sortie est borné par $N$.

Les points de $\bigcap_{i=-(N+1)}^{N+1} X^{i}(R)$ ne sortent pas de $M$, positivement et négativement avant un temps $N+1$, donc sont disjoints de l'orbite de $C$. Le saturé de la partition $\mathcal{R}^{\prime}$ dont les rectangles sont les composantes connexes de $\bigcap_{i=-(N+1)}^{N+1} X^{i}(R)$ est donc disjoint de $C$.

Alors d'après le corollaire précédent, $C$ borde sur $\partial_{1} M$ un disque disjoint $\operatorname{de} \operatorname{Sat}\left(\mathcal{R}^{\prime}\right)$ et, a fortiori, de $W^{s}(K)$.

On a ainsi achevé la démonstration de 0.1 et de 0.2 .

La proposition 3.8 a le corollaire important suivant :

CoRollaire 3.14. - Le nombre de composantes connexes de la surface de sortie $S$ associé au type géométrique $T$ ainsi que le genre de chacune de chacune de ces composantes connexes ne dépend que du germe du champ $X$ le long de $K$ et pas du type géométrique précis $T$.

Démonstration. - Rappelons d'abord que le théorème principal de [1], que nous avons énoncé dans l'introduction, affirme qu'il existe un unique 
modèle associé au germe d'un champ de vecteur le long d'un ensemble selle saturé.

D'après la proposition 3.8 , le bord de sortie du modèle est obtenu en recollant un disque sur chaque composante du bord de $S$. Chaque composante de $S$ correspond donc à une composante du bord de sortie du modèle de même genre.

Nous obtenons ainsi des invariants qui nous donnerons à la partie suivante des obstructions à être un germe de suspension d'ensemble selle saturé de difféomorphisme de surface.

\section{Champs de vecteurs et suspensions de difféomorphismes}

Un ensemble selle d'un champ de vecteurs en dimension 3 possède toujours un voisinage en restriction auquel le champ est équivalent à la suspension de la restriction d'un difféomorphisme de surface compacte à un voisinage d'un ensemble basique hyperbolique (basic set en anglais), c'est à dire d'un ensemble hyperbolique maximal invariant d'un de ses voisinages. En effet :

- Rappelons que l'existence de transversales locales et de partitions de Markov par des rectangles disjoints implique que tout ensemble selle est topologiquement équivalent à la suspension d'un sous-shift de type fini (voir [5]).

- Choisissons une transversale locale $\Sigma$ et une partition de Markov $\mathcal{R}$ dans $\Sigma$. L'application de premier retour $f$ sur $\Sigma$ est bien définie sur l'union des rectangles $R$; on peut inclure $R \cup f(R)$ dans une surface compacte $S$. Le difféomorphisme $f$ est défini d'une famille de $n$ rectangles disjoints de $S$ sur une famille de $n$ rectangles disjoints de $S$, on peut donc le compléter en un difféomorphisme de $S$; le maximal invariant de $R$ est alors un ensemble basique.

Nous nous intéressons ici à un point de vue global :

$\left.{ }^{*}\right)$ Un champ de vecteurs est-il toujours, en restriction à un certain voisinage invariant d'un ensemble selle saturé, topologiquement équivalent à la suspension d'un difféomorphisme de Smale de surface au voisinage d'un ensemble selle saturé ?

Dans la construction du difféomorphisme ci-dessus, l'ensemble basique peut ne pas être une pièce basique (une pièce basique est saturée). En effet, P. Blanchard et J. Franks ont montré (voir [3]) que certains sous-shifts de type finis n'étaient pas associés à des pièces basiques de difféomorphismes 
de surfaces. Dans le même esprit, [4] donne des obstructions à ce que certains types géométriques soient réalisables pour les difféomorphismes, c'est à dire correspondent à des partitions de Markov d'ensembles saturés de difféomorphismes de Smale de surfaces compactes.

Cependant, les obstructions de [3] et [4] ne répondent pas à notre problème $\left(^{*}\right)$ : deux choix de transversales du même ensemble selle donne lieu à des types géométriques et même à des sous-shifts différents. Un des types géométriques (ou des sous-shifts) peut être réalisable pour les difféomorphismes et pas l'autre, ce que montre l'exemple suivant.

\section{Exemple de deux types géométriques, de même modèle, l'un réalisable, l'autre pas :}

Considérons la partition à deux rectangles de la suspension du fer à cheval usuel dont les deux rectangles sont les sous-rectangles horizontaux de la partition de la figure 11 et détriplons le rectangle portant le point fixe coin (le dédoublement est une des deux opérations décrites dans [11] et engendrant la "flow-equivalence" ; le détriplement est défini de même) : on obtient la partition décrite à la figure 11 . On note $T$ le type géométrique de la nouvelle partition. Le champ correspondant est bien sûr la suspension du fer à cheval. Cependant :

Proposition 4.1. - Le type $T$ n'est pas réalisable pour les difféomorphismes.

Démonstration. - Supposons $T$ réalisé par une partition d'un ensemble selle saturé $K$ d'un difféomorphisme $f$ de surface compacte.

L'orbite coin de la suspension du fer à cheval engendre pour $T$ une orbite coin de période 3 de $K$. Chacun des points de cette orbite possède une séparatrice stable libre, c'est-à-dire disjointe de l'ensemble selle $K$. L'autre séparatrice stable est une séparatrice bord (elle n'est accumulée par $K$ que d'un seul côté) non libre.

En itérant 7 fois la partition de Markov (voir la figure 11), on voit que les trois séparatrices bords non libres sont deux à deux couplées, c'est-àdire deux à deux jointes par des segments de variété instable d'intérieurs disjoints de $K$; cette configuration n'est pas possible pour un ensemble selle saturé de difféomorphisme de surface compacte d'après [4, corollaire 2.4.3].

Nous voulons maintenant répondre négativement au problème $\left({ }^{*}\right)$ et donc décrire des exemples d'ensembles selles saturés de champs de vecteurs 

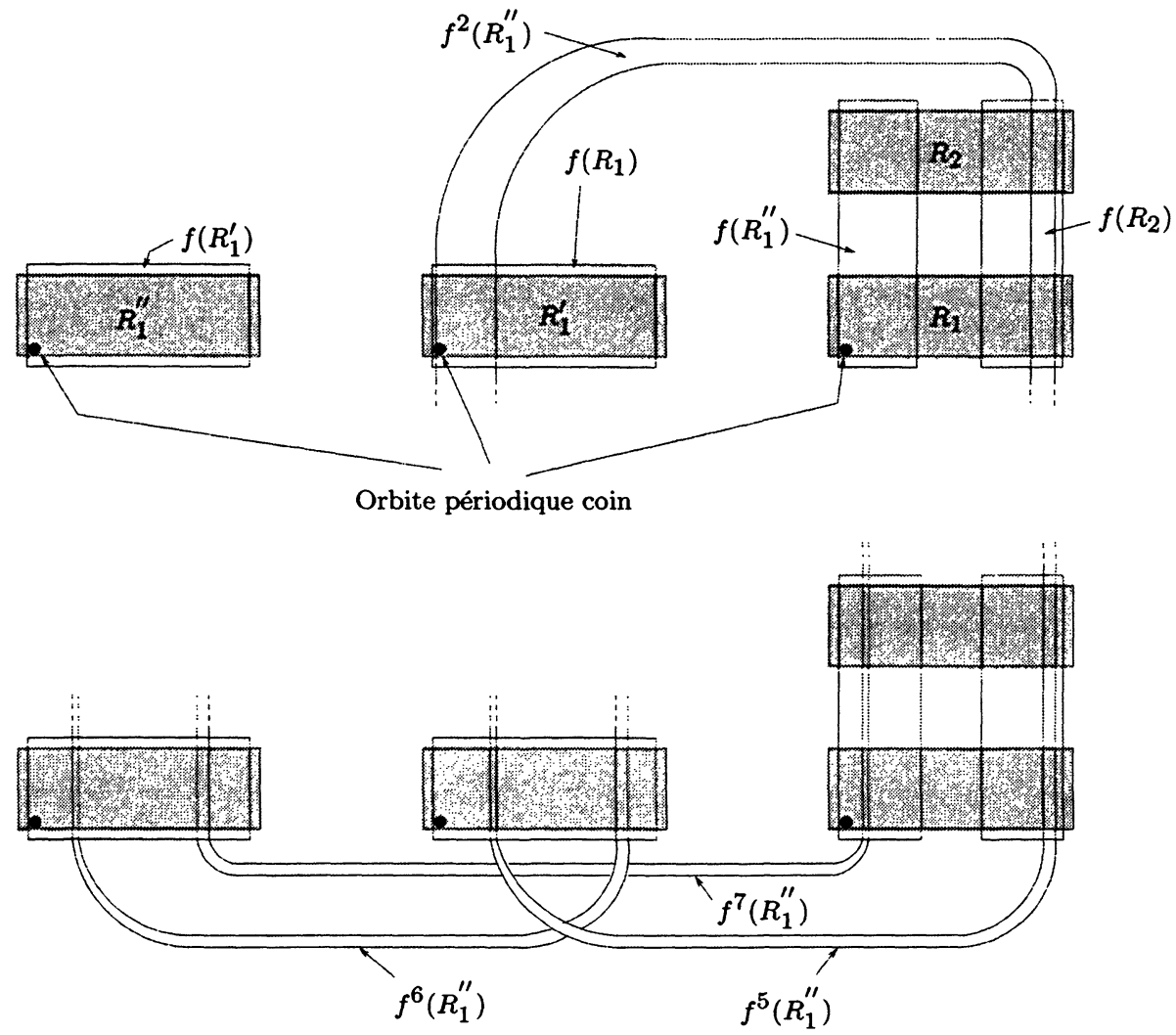

Fig. 11 La partition de Markov obtenue en détriplant le rectangle inférieur du fer à cheval et son itération partielle

en dimension 3 dont le germe n'est pas celui d'un ensemble selle saturé de suspension de difféomorphisme de surface compacte.

Proposition 4.2. - Soit $K$ un ensemble selle saturé d'un flot de Smale $X$ sur une variété compacte orientable de dimension 3 dont le germe est le même que celui de la suspension d'un difféomorphisme de Smale de surface compacte le long d'un ensemble selle saturé.

Alors toute composante du bord d'entrée ou de sortie du modèle de $[X, K]$ est de genre inférieur ou égal à 1 (c'est une sphère ou un tore).

Démonstration. - Tout ensemble hyperbolique saturé $K$ d'un difféomorphisme de surface $f_{0}$ possède un voisinage compact $U$ dont il est le 
maximal invariant tel que le saturé $\bigcup_{\mathbb{Z}} f_{0}^{n}(U)$ est homéomorphe à une surface compacte $S$ privée d'un nombre fini de points et tel que l'on peut compléter $f_{0}$ sur $S$ en un difféomorphisme $f$ de Smale en rajoutant un nombre fini de sources et de puits (voir, par exemple, [4, chapitre 1]) .

La suspension de $f$ donne lieu à une variété $\mathcal{M}$ de dimension 3. En ôtant à $\mathcal{M}$ un tore solide, à bord transverse au flot, autour de chaque orbite puits ou source, on obtient une variété $M$ compacte à bord munie d'un champ $X$ transverse au bord dont le maximal invariant est la suspension de $K$. C'est ce que dans [1], nous avons appelé voisinage filtrant de la suspension de $K$. Chacune des composantes des bords d'entrée et de sortie de ce voisinage filtrant est par construction un tore.

Dans [1], on construit le modèle de $[X, K]$ à partir de $M$ en via des chirurgies qui diminuent toujours le genre des bords d'entrée et de sortie. Toute composante du bord du modèle est donc de genre inférieur ou égal à 1 .

\section{Exemple d'ensemble selle saturé qui n'est pas une suspension :}

D'après le corollaire 3.14 et la proposition 4.2 , pour donner des exemples d'ensembles selles saturés dont le germe n'est pas celui d'une suspension le long d'un ensemble selle saturé, il suffit de donner des types géométriques donnant lieu à une surface de sortie $S$ dont une composante est de genre plus grand que 2. La figure 12 décrit un type géométrique donnant lieu à une surface de sortie de genre 2 et montre qu'il est très facile de construire d'autres exemples de tels types géométriques.

Le genre de $S$ n'est pas la seule obstruction à ce que le germe d'un flot le long d'un ensemble selle saturé soit celui d'une suspension de difféomorphisme de surface compacte le long d'un ensemble selle saturé. Le genre de $S$ donne lieu à l'obstruction la plus "pratique" car provenant d'un invariant calculable. Voici cependant un exemple qui utilise une obstruction plus fine et qui nous a paru surprenant.

Exemple de type géométrique $T$ qui est une "suspension" et tel que $T^{3}$ n'est pas une suspension :

Nous allons juste considérer le type $T$ défini ci-dessus à la figure 11 (partition à deux rectangles du fer à cheval dont on a détriplé le rectangle inférieur). Le type $T$ engendre, par construction, la suspension du fer à cheval usuel. Notons $\mathcal{R}$ une partition de Markov essentielle de type $T$ et $P$ l'application de premier retour sur l'union des rectangles de $\mathcal{R}$. Alors $\mathcal{R}$ est une partition de Markov pour $P^{3}$; notons $T^{3}$ sont type géométrique. 


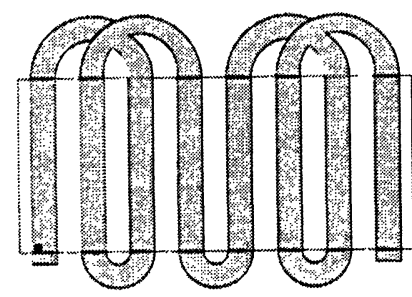

Partition, à 1 rectangle, de type géométrique $T$

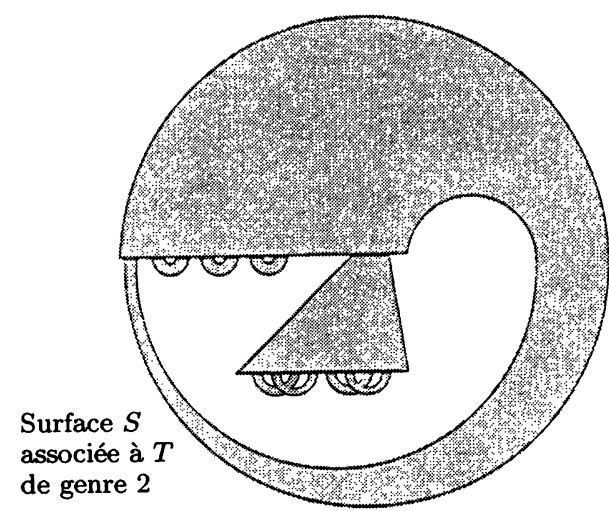

Fig. 12 Une partition à 1 rectangle et la surface de sortie $S$, de genre 2, associée à son type géométrique

Proposition 4.3. - Le type $T^{3}$ n'est pas le type géométrique d'une suspension.

Une démonstration précise serait inutilement longue et technique alors que les idées de la preuve sont simples; nous n'en donnerons que les grandes lignes.

Idée de la démonstration. - Reprenons la démonstration de la proposition 4.2 : la même idée montre en fait que, pour un flot obtenu comme suspension, tout ensemble selle saturé admet un voisinage filtrant dont le bord est formé de tores sur lesquels toute séparatrice libre dessine un cercle non homologue à zéro. En conséquence :

Soit $T_{0}$ le type géométrique d'une partition de Markov d'un ensemble selle saturé $K$ d'une suspension $X$ d'un difféomorphisme de surface compacte. Soit $(\tilde{M}, \tilde{X})$ le modèle de $[X, K]$ et $\partial_{2} M$ le bord de sortie de $\tilde{M}$. Alors il existe un voisinage de la lamination $W^{u}(K) \cap \partial_{2} M$ qui est plongeable dans une union de tores disjoints où le plongement de toute feuille compacte est non homologue à zéro.

Notons $P^{3}$ l'application de premier retour sur une partition essentielle de type géométrique $T^{3}$. Cette application possède 3 points fixes coins. Les séparatrices instables libre de ces points dessinent trois cercles sur le bord de sortie du modèle. Ces cercles correspondant à des orbites périodiques coins, il ne sont accumulés que d'un côté par des feuilles de la lamination. On peut voir que, pour deux quelconques de ces trois cercles, il existe une feuille 
compacte spiralant de l'une à l'autre. La raison en est que les séparatrices stables non libres des trois points périodiques sont deux à deux couplées (voir la démonstration de la proposition 4.1). On obtient alors une lamination partiellement décrite à la figure 13.

Il est impossible de plonger trois feuilles compactes ainsi couplées dans un tore chacune étant non homologue à zéro. En effet, deux quelconques des trois feuilles compactes découperaient alors une couronne dans le tore, l'âme de cette couronne étant constituée de la troisième feuille compacte. L'existence de deux feuilles spiralant de l'âme vers chacun des bords de la couronne implique que l'âme est accumulée de ses deux côtés. Cette contradiction termine la démonstration.

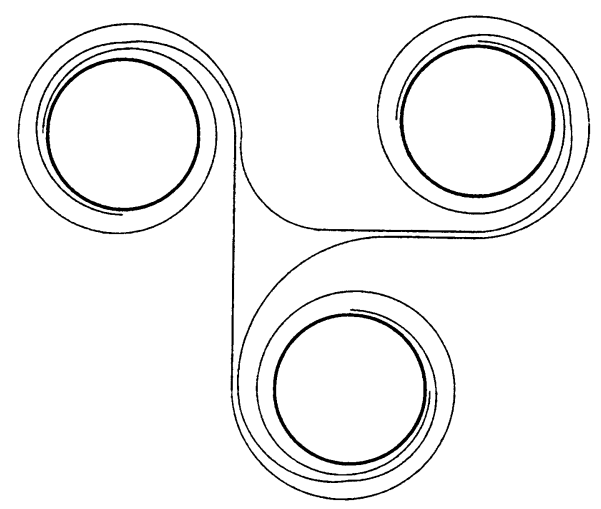

Fig. 13 Quelques feuilles de la lamination induite par la variété instable sur un bord de sortie pour le type $T^{3}$

Remarque. - Ici, la surface de sortie $S$ associée à $T^{3}$ est de genre nul.

\section{Bibliographie}

[1] Beguin (F.) et Bonatti (C.). - Flots de Smale en dimension 3 : présentation finie de voisinages invariants d'ensembles selles, à paraître à Topology.

[2] BiRman (J.S.) et WiLliams (R.F.). - Knotted periodic orbits in dynamical systems $I I$ : knots holders for fibered knots. Contemporary mathematics, Volume 20, 1983.

[3] Blanchard (P.) et Franks (J.). - An obstruction to the existence of certain dynamics on surfaces. Ergodic theory and Dynamical systems 1, pages 255-260, 1981.

[4] Bonatti (C.) et LANGevin (R.) avec la collaboration de E. Jeandenans. Difféomorphismes de Smale des surfaces. Astérisque, vol. 250, 1998. 


\section{F. Béguin, C. Bonatti et J.L. Vieitez}

[5] Bowen (R.). - One dimensional hyperbolic sets for flows. Journal of Differential Equations 12, pages 173-179, 1972.

[6] Franks (J.). - Symbolic dynamics in flows on three manifolds. Transactions of the American Mathematical Society 279, volume 1, pages 231-236, 1983.

[7] Ghrist (R.W.), Holmes (P.J.) et Sullivan (M.C.). - Knots and Links in ThreeDimesnional Flows. Lect. Note in Math., vol. 1654, Springer-Verlag, 1997.

[8] HAYASHI (S.). - Connecting invariant manifolds and the solution of the $C^{1}$-stability and $\Omega$-stability conjectures for flows. Annals of mathematics 145, pages 81-137, 1997.

[9] Hirsch (M.), Pugh (C.) et Shub (M.). - Invariant manifolds. Lectures Notes in Mathematics 583, Springer-Verlag, 1977.

[10] Jeandenans (E.). - Difféomorphismes hyperboliques des surfaces et combinatoire des partitions de Markov. Thèse de doctorat de l'Université de Bourgogne, 1996.

[11] PARRY (W.) and Sullivan (D.). - A topological invariant for flows on onedimensional spaces. Topology 14, pages 297-299, 1975.

[12] Pugh (C.) et Shub (M.). - Suspending subshifts. In Contributions to geometry and Analysis, C. Percelli and R. Sackester editors, John Hopkins University Press, 1981.

[13] Robinson (C.). - Structural stability of $C^{1}$ flows. Dynamical systems (Warwick, 1974), Lecture Notes in Mathematics 468, pages 262-277, Springer Verlag, 1975. 